\title{
Uncertainty and Sensitivity Analysis of Significant Parameters for Superlarge Diameter Shield Excavation
}

\author{
Elton J. Chen $\mathbb{D}^{1,2}$ Yang-Yang Chen,, ${ }^{1,2}$ Lin-Chun Wei, ${ }^{3}$ and Han-Bin Luo ${ }^{1,2}$ \\ ${ }^{1}$ School of Civil \& Hydraulic Engineering, Huazhong University of Science and Technology, Wuhan 430074, China \\ ${ }^{2}$ Center for Virtual, Safe and Automated Construction of Hubei Province (ViSAC), Wuhan 430074, China \\ ${ }^{3}$ Shanghai Tunnel Engineering Co., Ltd., Shanghai 200032, China
}

Correspondence should be addressed to Elton J. Chen; eltonjchen@hust.edu.cn

Received 2 October 2020; Revised 1 December 2020; Accepted 10 February 2021; Published 23 February 2021

Academic Editor: Wenping Gong

Copyright ( 92021 Elton J. Chen et al. This is an open access article distributed under the Creative Commons Attribution License, which permits unrestricted use, distribution, and reproduction in any medium, provided the original work is properly cited.

Excavation of a superlarge diameter tunnel by tunnel boring machine (TBM) is different from that of a shield tunnel with normal dimension, in which the control system of the superlarge TBM is very complicated and difficult to operate. Hence, it is very important to focus on the control and management of significant parameters to ensure excavation stability under uncertainty. In this paper, we (i) utilize a BIM-based big data platform (BIM-BDP) to manage the essential construction data of tunnel project in digital format; (ii) adopt the global sensitivity analysis (SA) to recognize significant parameters for shield excavation based on polynomial chaos expansion (PCE)-extended Fourier amplitude sensitivity test (eFAST) model; and (iii) employ the uncertainty analysis (UA) to discover the correlation between significant parameters from the data of the BIM-BDP. This research contributes to (i) the body of knowledge of proposing a more appropriate research methodology that can cope with aleatory and epistemic uncertainty and support uncertainty and sensitivity analysis (UA/SA) processes based on data from BIM-BDP and (ii) the state of practice by providing a data-driven surrogate model to simulate system behaviors of shield excavation with high reliability and to reduce dependency on domain experts. Here, we pay close attention to the most influential parameters that require priority parameter control, which can help administrators optimize the management of shield parameters during tunnel excavation.

\section{Introduction}

Tunnel boring machine (TBM) is the main tool for urban tunnel excavation due to its high excavation efficiency, high safety, environmental friendliness, and high cost-effectiveness [1]. In order to guarantee the safety and efficiency of tunnel excavation, the administrators need to (i) record and store real-time monitoring data during TBM tunneling, including hydrological, geological, and TBM parameters; (ii) identify the influential and noninfluential parameters for excavation stability based on the monitoring data; (iii) control and manage influential parameters under system uncertainty. To avoid excavation instability, it is very meaningful to manage and analyze the construction parameters [2-8]. Building Information Modeling (BIM) enables the design and analysis of complex, multidisciplinary systems as often encountered in infrastructure projects and, in particular, tunneling [9].
Reasonable control of TBM is considered as a complicated process, in which it is very difficult to rigorously analyze interactive relationship between construction parameters due to the uncertainty of heterogeneous soil and complex surroundings. The uncertainty and sensitivity analysis (UA/SA), which helps researchers better understand the relative importance of each parameter $[10,11]$, of shield machine construction parameters is an important research direction for parameters control. Understanding the relative "sensitivity" of parameters can aid in the development of better monitoring strategies and experiment design, for example, indicating the priority and amount of parameters to be controlled. In the SA methods, the global sensitivity analysis (GSA) can perceive and distinguish the influence magnitude of parameters associated with the excavation stability to help reduce the cognitive uncertainty caused by the limitation of human cognition and uncertain factors, for 
example, incomplete information. Hence, GSA provides guidance for identifying parameters whose information should be collected to enhance the system reliability. The extended Fourier amplitude sensitivity test (eFAST) method is a global and quantitative GSA algorithm that can be applied to complex nonlinear and nonmonotonic models [12-14]. The key idea of eFAST is that from one simulation run to another, all factors fluctuate around their nominal values [15]. The importance of the factor is determined by analyzing the Fourier decomposition of the model response. The eFAST method has been utilized in this paper to investigate the complicated and uncertain interaction between the construction parameters as well as the excavation stability in the SRHT project.

This research contributes to (i) developing a framework of UA/SA for investigating the interactive relationship between construction parameters under system uncertainty; (ii) proposing a noval polynomial chaos expansion-extended Fourier amplitude sensitivity test (PCE-eFAST) model for GSA with high accuracy and reliability; (iii) exploring the reasonable sample size for PCE model and investigating the limitation of eFAST method to get a more convincing result.

The remainder of this paper is organized as follows. First, the literature review of parameters analysis for superlarge diameter shield excavation is briefly presented. Second, Section 3 shows the construction of the BIM-BDP, which includes four modules (i.e., data sensing, data storage, data analysis, and data presentation). Third, the global uncertainty and sensitivity analysis process based on BIM-BDP data is exhibited in Section 4, with three steps (i.e., generating data according to the input-output index system, running the surrogate model, and performing GUA and GSA by PCEeFAST method). Fourth, the UA/SA results are revealed in Section 5. Fifth, we discuss the limitation of the eFAST method and this study. The final section summarizes the main observations of the study and lists future research directions.

\section{Literature Review}

2.1. Parameters Analysis for Superlarge Diameter Shield Excavation. TBM is extremely sensitive to geological changes and excessively dependent on the operator's own experience [16]. BIM for decision making during the life cycle of infrastructure projects is a vital tool for the analysis of complex, integrated, multidisciplinary systems [9]. The BIM mainly contains both geometric and nongeometric data, and a strong link should be formed between the visual model and database [17]. For underground projects, [12] established a BIM cloud-based safety risk knowledge database to sort out all kinds of safety risk knowledge. A study integrated WNS and BIM to carry out long-term underground environment detection and monitoring, and the collected data can be preserved in a database for analysis and management [18]. As for the integrated database, data incompatibility is the most significant problem [19]. One of the most valuable assets in any database is the content itself [20]. However, as far as the literature is concerned, there are relatively few studies that make full use of and analyze the data, especially in the shield tunnel project.

TBM is a kind of very complex and comprehensive equipment and is composed of a variety of systems. Although TBM can provide hundreds of shield machine operating parameters, the TBM operator still has an inadequate awareness of geological conditions and shield tunneling machine status at present and of whether such shield machine operation status corresponds to such geological conditions. Therefore, there are still many problems involved in the operation of the shield machine during the TBM construction, such as how to evaluate rock parameters in real-time based on equipment parameters and how to use the evaluation results as the basis for decision making and optimization to achieve scientific control and management of shield parameters for excavation stability.

\subsection{Polynomial Chaos Expansion (PCE). The random} spectrum method [21] is a new technique for establishing a surrogate model. The method has a multidimensional random response surface in the sample space and a large number of response samples. The most famous random spectrum method is polynomial chaos extension (PCE). The authors in [22] proposed an initial Hermite polynomial chaos method, which uses normal random variables. The authors in [23] proposed a generalized PCE method and extended the method to non-normal random variables (e.g., lognormal, Weibull, and beta). Besides, after further development, generalized PCE can already use non-global smoothing polynomial basis functions. The Hermite polynomial chaos method for moving and windowing can estimate a small activation probability and improve the accuracy of the response surface [24].

PCE has many advantages over other surrogate models [25]. This method can produce highly accurate results, and it has proven to have significant computational efficiency in the expansion of large-scale systems [26]. The use of nonintrusive PCE in the quantification of uncertainty has received wide attention. The method has been applied as well in solving fluid dynamics, circuit simulation, environment, and sound field. The basic idea is to approximate the stochastic process by the sum of orthogonal polynomial chaos with independent random variables. The key step is to determine the coefficients of each polynomial. The model responses are characterized by a multivariate polynomial whose distribution relative to the input variables is orthogonal. In this case, characterizing the probability density function $(\mathrm{PDF})$ is equivalent to evaluating the polynomial chaos coefficients.

2.3. Global Uncertainty and Sensitivity Analysis. The fact that SA should be a fundamental part of any analysis that involves the assessment and propagation of uncertainty is underlined in [27]. SA of a model aims at quantifying the relative importance of each input parameter [28]. In particular, techniques for GSA have become an accepted standard for the evaluation of the impact and interactions of uncertain inputs in complex environmental effects [29-31]. 
Uncertainty is a physical quantity that characterizes how reliable a random variable is and refers to situations involving imperfect and unknown information. It appears in a partially observable and/or random environment, which is suitable for the prediction of future events [32]. The GSA considers the output behavior of the model over the full domain of uncertain inputs; specifically, this implies that the full distribution of each input parameter should be evaluated and that the importance of each input should be evaluated across the domain of all other parameters [33,34].

The main methods for performing GSA include variance-based methods, regression methods, and Morris screening methods. Among these GSA methods, the variance-based methods have restrictions on the output distribution. Regression methods require knowledge of the input-output relationship form. Morris screening methods cannot compare the magnitude of sensitivity. Only variancebased methods can explore the whole space of the uncertain inputs [35]. The inputs are usually carried out via Monte Carlo (MC) sampling, and the basic idea of SA based on variance is to analyze the effect of input on output variance.

A common type of GSA is the variance-based method, which operates by apportioning the variance of the model's output into different sources of variation in the inputs $[15,36]$. More specifically, it quantifies the sensitivity of a particular input (the percentage of the total variability in the output attributed to the changes in that input) by averaging over other inputs rather than fixing them at specific values. The Fourier amplitude sensitivity test (FAST) was one of the variance-based methods [37]. The classical FAST method uses spectral analysis to apportion the variance, after first exploring the input space using sinusoidal functions of different frequencies for each input factor or dimension [38].

The FAST is a variance-based method that is used to calculate the main sensitivity index (MSI) by scanning the parameter space with periodic functions such that the entire sample space can be analyzed [31]. A multidimensional integration is reduced to $1 \mathrm{D}$ integration along with a curve by associating each variable with a sampling frequency of the system in the Fourier transform space, which greatly improves the sampling efficiency [39, 40]. Then, Saltelli et al. [12] extended the FAST method to perform the total sensitivity index (TSI), which is called eFAST method. This eFAST method, combining the benefits of the FAST and Sobol's method, is highly efficient and may be used to analyze the interactive relationship between various parameters.

In the present work, the MC method is used to evaluate the effect of each input error on the output [27]. Firstly, the PCE-eFAST model is established. Then, the statistical characteristics of the parameters are calculated by performing a large amount of random sampling on the parameters in the model. Finally, the approximate value of the solution is obtained. It is concluded that the necessary precautions should be taken to reduce the impact of changes in these parameters on the parameters of the shield machine equipment and to minimize errors or disturbances.

\section{The Architecture of the BIM-BDP}

TBM operations are greatly affected by geological conditions and heavily rely on the operator's own subjectivity. In the case that external environment changes, it is difficult for TBM to perceive the status of geotechnical and shield machines in time to make scientific judgments to guide construction, leading to frequent occurrence of engineering accidents during TBM tunneling, such as ground settlement and cutter head damage, and thus resulting in huge economic losses. Therefore, it is necessary to remotely monitor and control different types and brands of shield machines to collect construction information to guide construction. In order to realize the scientific and efficient construction management of the shield tunnel, a shield construction information management system integrating project management, data analysis, and construction decision support is particularly important.

In this study, a UA/SA method is proposed for data analysis and decision making during tunnel excavating. To improve its prediction accuracy, a variety of sensors are employed, covering both shield machine and stratum. Furthermore, in order to facilitate the data collection, processing, analysis, and presentation, an BIM-BDP is developed. The entire system is structurally divided into a sensing module, a storage module, an analysis module, and a presentation module, as shown in Figure 1. The sensing module and storage module are used to collect and store the shield machine data of the tunnel project, and the analysis module is used to analyze shield machine parameters. The presentation module exhibits the BIM-BDP through the browser client and realizes the functions of visualizing the shield parameter data and applying the platform.

The TBM construction data sensing layer needs to collect real-time information related to TBM operation and main control parameters. This real-time information should be related to the whole process of shield tunnel construction, types, and stages. This information is divided into three categories according to engineering objects: shield machine (construction equipment), geological space (Earth layer information), and tunnel structure (engineering entity). The shield machine information mainly includes its overall geometry, performance parameter description, and key construction parameters of each subsystem of the shield; the tunnel structure includes tunnel linear design, functional design parameters, segment ring design parameters, and tunnel forming quality parameters; geological space includes geological information such as soil parameters, soil stratification, and hydrological information (e.g., groundwater level, head, and seepage). This information plays an important role in supporting the progress, quality, cost, and safety management of the shield tunnel project. The information classification and storage methods are shown in Figure 1.

The data stored by the module includes real-time data of the shield machine, manual data, and BIM 3D model data. The three types of data are stored in the database server and 


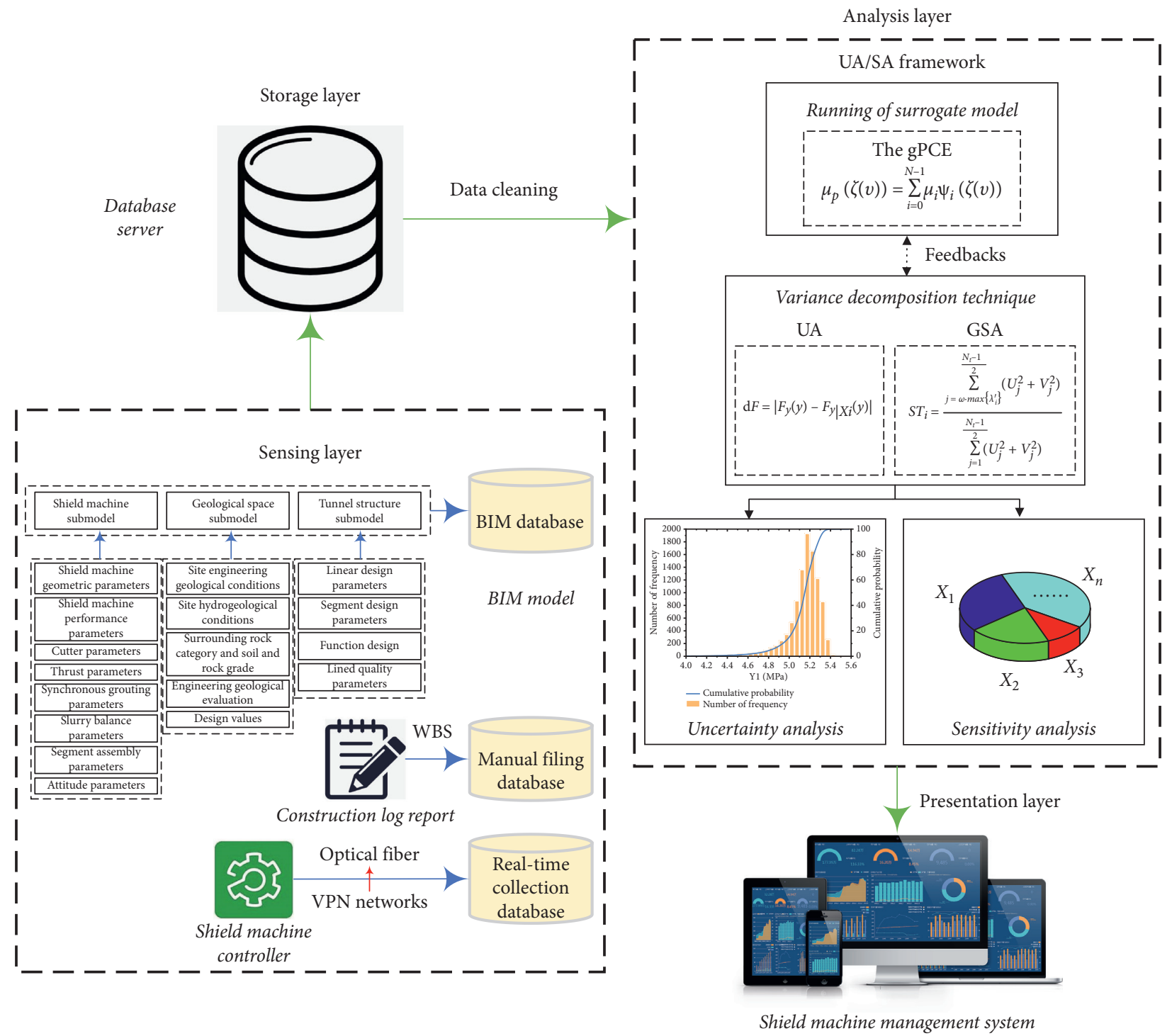

FIgURE 1: The architecture of the BIM-BDP.

establish a request and response relationship with the web server. The web server then establishes a request with the browser. The browser sends a request to the web server in an HTTP format to access the cloud database. After the web server accepts the server request, the request is automatically converted into a SQL query statement and passed to the database server. After receiving the request, the database server needs to verify its legitimacy, perform data processing, and then return the processed structure to the web server. The web server then converts the obtained result into an HTML document form and displays a friendly web page through a browser.

The analysis module is the core of the BIM-BDP, which embeds the sensitivity and uncertainty analysis algorithm. The UA/SA makes use of the previously developed BIMBDP that collects the excavating information of SRHT. Numerous methods, based on either deterministic or statistical concepts, have been proposed for performing UA/ SA. Most of the existing methods require explicit expressions (equations or functions) between the inputs and outputs. The common way of quantifying uncertainty is estimating the PDF [41] and the cumulative distribution function (CDF) $[31,42]$. It is considered that the parameters or the interaction between the input parameters causes the variance of the model output; thus, PDF and CDF can reflect the sensitivity of the model output to the input parameters $[43,44]$. Therefore, the coupling among the parameters and the total variance parameter can be analyzed by GSA [45]. For the GSA study, the surrogate model (also known as metamodel) is commonly employed to represent the uncertainty in the output and calculate the mean and standard deviation [46]. We adopt the generalized polynomial chaos expansion (gPCE) method [47] to construct a computationally cheaper surrogate to approximate the mapping between input parameters and output parameters. Then, the eFAST method is adopted to obtain the total-order sensitivity index, which takes into account the main effect of a single parameter on the output and the interaction between 
the parameters and describes the total influence of a parameter on the output.

The data presentation module is based on the browser display and uses the Silverlight plug-in to develop an interactive interface to increase system interactivity. The module can realize the visualization of the BIM and its associated files, full network visualization, and real-time key parameters of the shield machine. In addition, the system provides multiple data analysis tools to support construction decision making. Besides, users can easily find the construction time and material consumption of any component by only selecting it on the BIM. For example, if any lining ring is selected, the construction time and material consumption of the ring can be counted. By querying the historical data and exporting the data, the excavation surface stability analysis and GSA can be further developed based on the data.

In order to better collect, transmit, store, and use these data, the platform supports multiuser access and remote login monitoring via the Internet, while reducing the dependence on front-end hardware and software, and its maintenance and upgrade are easy. The system is built using the browser/server $(\mathrm{B} / \mathrm{S})$ architecture and uses Java Development Kit (JDK) as the development platform, MyEclipse as the development tool, Java as the programming language, and SQL Server 2008 as the database.

\section{The UA/SA Framework}

The global scheme that describes the required different steps for the computation of partial variances is presented in Figure 2. The proposed framework (as given in Figure 2) consists of three main steps. In Step 1: generating data based on the input-output index system, we confirm the inputs and outputs from the BIM-BDP and generate the input data by the same "level of uncertainty" according to the same coefficient of variation (COV). In Step 2: running the surrogate model, surrogate models are built for the response and coupling variables, and then we can extract the output data by rerunning of the surrogate model. In Step 3: performing GUA and GSA by PCE-eFAST method, the uncertainty and sensitivity measures of inputs are estimated, and the input parameters include the MSIs and TSIs. In Sections 4.1-4.3, we explain the three steps in detail. For illustration, we use the BIM-BDP to verify the applicability of this method.

4.1. Step 1: Generating Data according to the Input-Output Index System. The parameters of BIM-BDP data can be sorted in to three categories: shield machine, tunnel structure, and geological space, including more than 900 groups of shield construction parameters as well as more than 30 construction monitoring parameters. However, due to the limited attention of the shield machine operator, it is impossible to monitor and control all these parameters simultaneously. Hence, some significant parameters, which have a prominent effect on the safety of shield tunneling, are considered as key control parameters for the shield machine operator.
Previous studies $[48,49]$ have primarily focused on the mechanical performance of the TBM in the construction phase, such as the maximum thrust force, permeability, and rotation speed of the cutter head. Berthoz et al. investigated the identification of pertinent control parameters to guarantee the safe advancement of the machine and the ground-supporting function of the cutting wheel, such as advancing speed, total volume loss, thrust effort, and torque on the cutter head [3]. Zou [50] indicated that a synchronous grouting technique can improve the stability of the surrounding rock and control the ground surface deformation in the shield tunnel construction process. Besides, based on construction experiences and previous studies $[14,51]$, the deviation angle and bubble chamber pressure are the main monitoring parameters for a shield machine operator to evaluate the stability of shield excavation. According to statistical analysis [52], it is found the attitude and position of the shield machine are mainly determined by the thrust cylinder and cutting wheel systems. Hence, some pertinent control parameters (as shown in Table 1) on the BIM-BDP are regarded as significant parameters for UA/SA study. Herein, the $x 1-x 10$ are the inputs and the $s 1$ $s 2$ are the outputs. In this study, the distribution of input parameters and output parameters is defined as follows: (i) Beta $(r, s, a, b): r>0, s>0$. The support of the distribution is given by the parameters $[a, b]$. The shape of the distribution is related to parameters $[r, s]$ and their ratio. (ii) Logistic $(\mu$, $s): \mu \in R, s>0$. The logistic distribution is similar to the normal distribution in shape but has heavier tails (higher kurtosis). (iii) Laplace $(\mu, b): \mu \in R, b>0$. Laplace distribution is also known as double exponential distribution, because it can be thought of as two exponential distributions (with an additional location parameter) spliced together back-to-back.

Determining the range and distribution of each input parameter is important in GSA study $[53,54]$, because it may affect the parameter TSIs and rank [55]. In general, two methods can be used to set the parameter ranges and distributions: (1) for those parameters with clear physical interpretations, their values should cover the entire physical range as much as possible; (2) if a parameter has no clear physical interpretation, one should refer to the literature or expert experience. In this study, all the parameters in the BIM-BDP have clear physical or geometric significance. The beta, logistic, and Laplace distributions have been adopted to fit the parameters. According to the fitting accuracy, the best fitting distribution and the parameter of each input have been summarized in Table 1 . Then, the inputs sets for UA/SA can be generated based on the best fitting distribution. Besides, scatter plots constitute an important class of visualization method. Scatter matrix plots are high-dimensional extensions of scatter plots, which can effectively show the pairwise relationship of multidimensional data. As shown in Figure 3, there is a high correlation between $x 4$ (advancing speed) and $x 9$ (average soil excavation volume with cutter rotation $360^{\circ}$ ). It is obvious that, with the increase in the advancing speed, the average soil excavation volume with cutter rotation $360^{\circ}$ also increases significantly. 

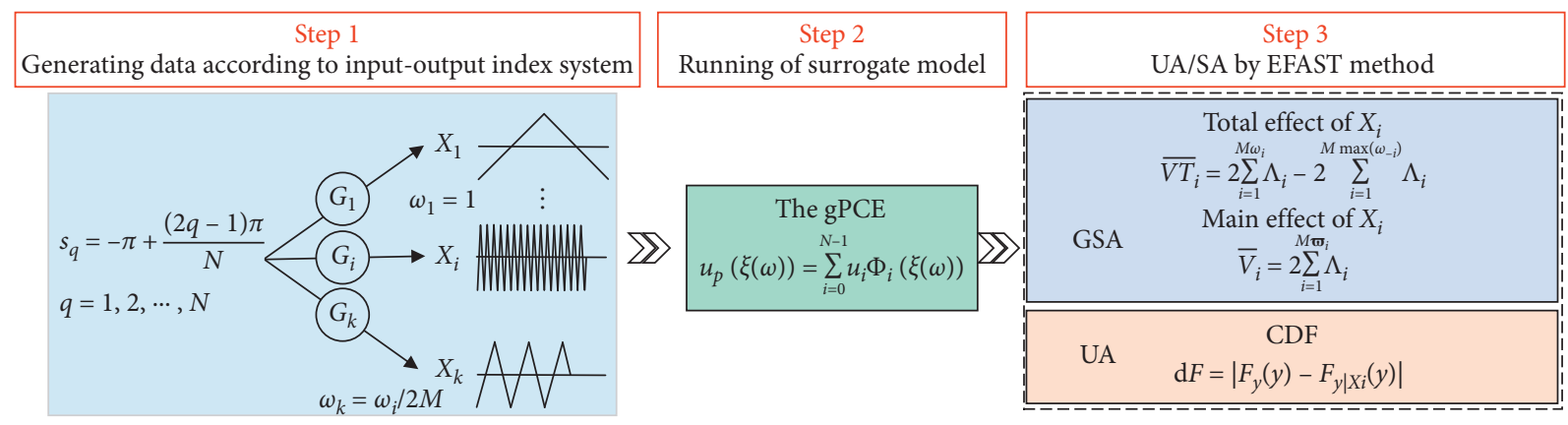

$k$ : number of parameters

$M$ : number of harmonics

$N$ : sampling size

Figure 2: The UA/SA scheme that describes the required different steps for the computation of partial variances.

4.2. Step 2: Running the Surrogate Model. The PCE model is popular for uncertainty propagation and GSA to determine the effect of input uncertainties on complex computational models [56, 57]. Currently, we use random variables to represent the uncertainty in the system. In this section, we introduce the basics of the gPCE [47] for stochastic uncertainty propagation.

We use a triplet $(\Omega, F, P)$ to represent a complete probability space, where the sample space $\Omega$ is the set of all possible outcomes, $F \subset 2^{\Omega}$ is the $\sigma$-algebra collection of all measurable events belonging to $\Omega$, and $P: F \longrightarrow[0,1]$ is a probability measure indicating the likelihood of occurrence of each event. Let $\zeta=\left\{\zeta_{1}(v), \zeta_{2}(v), \ldots, \zeta_{n}(v)\right\}: \Omega \longrightarrow \Xi \subseteq R^{n}$ be a set of uncorrelated random variables that represent the uncertainty in the system. Then, any second-order random variables $\mu(v) \in L(\Omega, P)$ (i.e., $\left.(\mu, \mu)=\|\mu\|_{\Omega}^{2}<\infty\right)$ can be represented as follows [28, 57]:

$$
\mu(\zeta(v))=\sum_{i=0}^{\infty} \widehat{\mu}_{i} \Psi_{i}(\zeta(v))
$$

where $\Psi_{i}$ 's were originally proposed by Wiener as Wiener-Hermite polynomial chaos (assuming that $\zeta$ has a Gaussian distribution) and later extended to generalized polynomial chaos (more classical orthogonal polynomials) by the Askey scheme [58]. The choice of polynomial chaos types depends on the distribution of random inputs. In the current work, we use Hermite polynomials for Gaussian random variables and Legendre polynomials for uniform random variables; the parameters $\mu_{i}$ 's are called the gPCE coefficients.

For the purpose of numerical calculation, the series is usually truncated up to polynomial order $p$ with $N$ terms $(N=(n+p) ! / n ! p !)$ to approximate the exact output $\mu(\zeta(v))$ :

$$
\mu_{p}(\zeta(v))=\sum_{i=0}^{N-1} \mu_{i} \Psi_{i}(\zeta(v))
$$

Based on the orthogonality of the polynomial basis, the gPCE coefficients can be calculated by projecting $\mu$ on each basis using the inner product:

$$
\mu_{i}=\frac{\left(\mu, \Psi_{i}\right)}{\left(\Psi_{i}, \Psi_{i}\right)}=\frac{1}{E\left[\Psi_{i}^{2}\right]} \int_{\Xi} \mu(\zeta) \Psi_{i}(\zeta) \rho(\zeta) \mathrm{d} \zeta
$$

where $\rho(\zeta)$ is the probability distribution of the variable $\zeta$, and the integration can be estimated using a quadrature rule:

$$
\mu_{i} \approx \widehat{\mu}_{i}=\frac{1}{E\left[\Psi_{i}^{2}\right]} \sum_{k=1}^{G} \mu\left(\zeta^{k}\right) \Psi_{i}\left(\zeta^{k}\right) \varepsilon^{k}, \quad i=0,1, \ldots, N-1
$$

where $\left\{\zeta^{k}, \varepsilon^{k}\right\}_{k=1}^{G}$ is a set of quadrature points and the corresponding weights. For low-dimensional problems, the use of tensor product quadrature may have significant efficiency; however, since the number of quadrature points increases exponentially as the dimension increases, it suffers from the "curse of dimensionality" for high-dimensional cases.

Once an accurate gPCE $\mu_{p}(\zeta)$ is constructed to approximate the random function $\mu(\zeta)$, one can obtain an analytical representation of the function $\mu$ in terms of $\zeta$. Therefore, all the statistical information of $\mu$ can be retrieved from the gPCE in a straightforward manner using their definitions directly. The $j$ th moment of $\mu$ for $j \in N$ is

$$
\begin{aligned}
E\left[(\mu-E[\mu])^{j}\right] & \approx E\left[\left(\mu_{p}-E\left[\mu_{p}\right]\right)^{j}\right] \\
& =\int_{-\infty}^{\infty}\left(\mu_{p}(\zeta)-E\left[\mu_{p}\right]\right)^{j} \rho(\zeta) \mathrm{d} \zeta,
\end{aligned}
$$

which can be calculated either analytically or with small computational effort. In particular, the first and second moments of $\mu$ can be obtained directly from the gPCE coefficients as follows. The expectation of $\mu$ only depends on the first coefficient: 
TABLE 1: The significant parameters on the BIM-BDP for UA/SA study.

\begin{tabular}{|c|c|c|c|c|c|}
\hline Type & Parameter & Unit & Distribution & Parameter values & Description \\
\hline \multirow{8}{*}{ Inputs } & $\begin{array}{l}\text { Maximum thrust force per } \\
\text { ring }(x 1)\end{array}$ & $\mathrm{kN}$ & Beta & $\begin{array}{c}{[2.64,3.12,4827.65} \\
12391.16]\end{array}$ & $\begin{array}{l}\text { The maximum thrust force needs to be } 1.5 \sim 2 \text { times } \\
\text { the total resistance. The total resistance mainly } \\
\text { includes the resistance of shield shell and } \\
\text { surrounding strata, the thrust resistance of knife disc } \\
\text { plate, the friction resistance between tube sheet and } \\
\text { shield tail, the penetration resistance of the stratum } \\
\text { through the notched ring, steering resistance, and } \\
\text { the drag resistance of the supporting trailer after } \\
\text { traction. }\end{array}$ \\
\hline & $\begin{array}{l}\text { Average synchronous } \\
\text { grouting pressure per ring } \\
\qquad(x 2)\end{array}$ & Bar & Beta & $\begin{array}{l}{[5.84,5.13,-13.86} \\
\quad 99.70]\end{array}$ & $\begin{array}{l}\text { Simultaneous grouting is a method of directly } \\
\text { injecting the slurry into the rear clearance of the } \\
\text { shield segment. The grouting pressure is required to } \\
\text { be greater than the sum of static water pressure and } \\
\text { soil pressure. }\end{array}$ \\
\hline & $\begin{array}{l}\text { Average synchronous } \\
\text { grouting volume per ring } \\
\qquad(x 3)\end{array}$ & $\mathrm{L} / \mathrm{min}$ & Logistic & {$[17.37,2.96]$} & $\begin{array}{l}\text { The synchronous grouting volume is the gap } \\
\text { between the cutting soil and the pipe wall in theory, } \\
\text { and, of course, factors such as rectifying deviation in } \\
\text { the advancing process of the shield, running } \\
\text { grouting (including diffusion to the stratum), and } \\
\text { grouting material shrinkage should be considered. }\end{array}$ \\
\hline & Advancing speed $(x 4)$ & $\mathrm{mm} /$ & Laplace & {$[7.27,3.41]$} & $\begin{array}{l}\text { The distance a shield machine can advance per unit } \\
\text { of time. }\end{array}$ \\
\hline & $\begin{array}{l}\text { Average grease weight of } \\
\text { shield tail per ring }(x 5)\end{array}$ & $\mathrm{L} / \mathrm{min}$ & Beta & $\begin{array}{c}{[2.54,2370650.02} \\
13.18,99990365.76]\end{array}$ & $\begin{array}{l}\text { A paste made by adding additives such as fiber, } \\
\text { modifier, and filler, mainly acting as a sealing, } \\
\text { waterproof, lubricating, and preservative. }\end{array}$ \\
\hline & $\begin{array}{l}\text { Average cutter rotation } \\
\text { speed per ring }(x 6)\end{array}$ & $\mathrm{r} / \mathrm{min}$ & Beta & $\begin{array}{l}{[1.56,1.13,-0.01} \\
1.47]\end{array}$ & $\begin{array}{l}\text { The linear velocity of the cutter rotating per unit } \\
\text { time divided by the value of its circumference. }\end{array}$ \\
\hline & $\begin{array}{l}\text { The ratio of intake/discharge } \\
\text { mud per ring }(x 7)\end{array}$ & - & Logistic & {$[0.01,0.01]$} & $\begin{array}{l}\text { The ratio of the volume of mud intake per unit of } \\
\text { time divided by the volume of mud discharged per } \\
\text { unit of time. }\end{array}$ \\
\hline & $\begin{array}{l}\text { Average cutter torque per } \\
\text { ring }(x 8)\end{array}$ & $\mathrm{MNm}$ & Beta & $\begin{array}{l}{[0.99,1.51,0.03} \\
25.83]\end{array}$ & $\begin{array}{l}\text { The cutter torque mainly includes cutting torque of } \\
\text { cutter disc, bearing torque formed by self-weight of } \\
\text { cutter disc, bearing torque formed by axial load of } \\
\text { cutter disc, friction torque of sealing device, friction } \\
\text { torque of blade front surface, friction counter- } \\
\text { torque of blade circumference surface, friction } \\
\text { torque of blade back, shear torque of opening groove } \\
\text { of cutter disc. }\end{array}$ \\
\hline
\end{tabular}

volume with cutter rotation $\mathrm{m}^{3} \quad$ Laplace $\quad[6.24,2.91] \quad$ The depth of the cutter wheel rotates into the soil. $360^{\circ}(x 9)$

Actual excavation volume per ring $(x 10)$

L Laplace

[343.97, 103.75]

The product of advance distance of shield machine and the cross section area of shield cutter.

The bubble chamber is located behind the mud

Average bubble chamber pressure per ring $(s 1)$

$\mathrm{MPa} \quad$ Beta

$[3.56,2.64,2.67$ 3.20]

Outputs

Deviation angle per ring $(s 2)$ o chamber, containing the mud, water, and air, and the pressure is the air pressure inside the bubble chamber.

The angle between the actual heading direction of the shield machine and the direction of the design

$$
E[\mu] \approx E\left[\mu_{p}\right] \approx \sum_{i=0}^{N-1} \widehat{\mu}_{i}\left(\Psi_{0}, \Psi_{i}\right)=\widehat{\mu}_{0}\left(\Psi_{0}, \Psi_{0}\right) .
$$

The variance $\sigma_{\mu}^{2}$ is obtained as a weighted sum of its squared gPCE coefficients:

$$
\sigma_{\mu}^{2} \approx \sigma_{\mu_{p}}^{2}=E\left[\left(\mu_{p}-E\left[\mu_{p}\right]\right)^{2}\right] \approx \sum_{i=1}^{N-1} \widehat{\mu}_{i}^{2}\left(\Psi_{i}, \Psi_{i}\right) .
$$

By gPCE method, we can quantify the uncertainty though the quantity of interest $g$ (propagated from the uncertainty in the inputs) efficiently using its stochastic moments. 


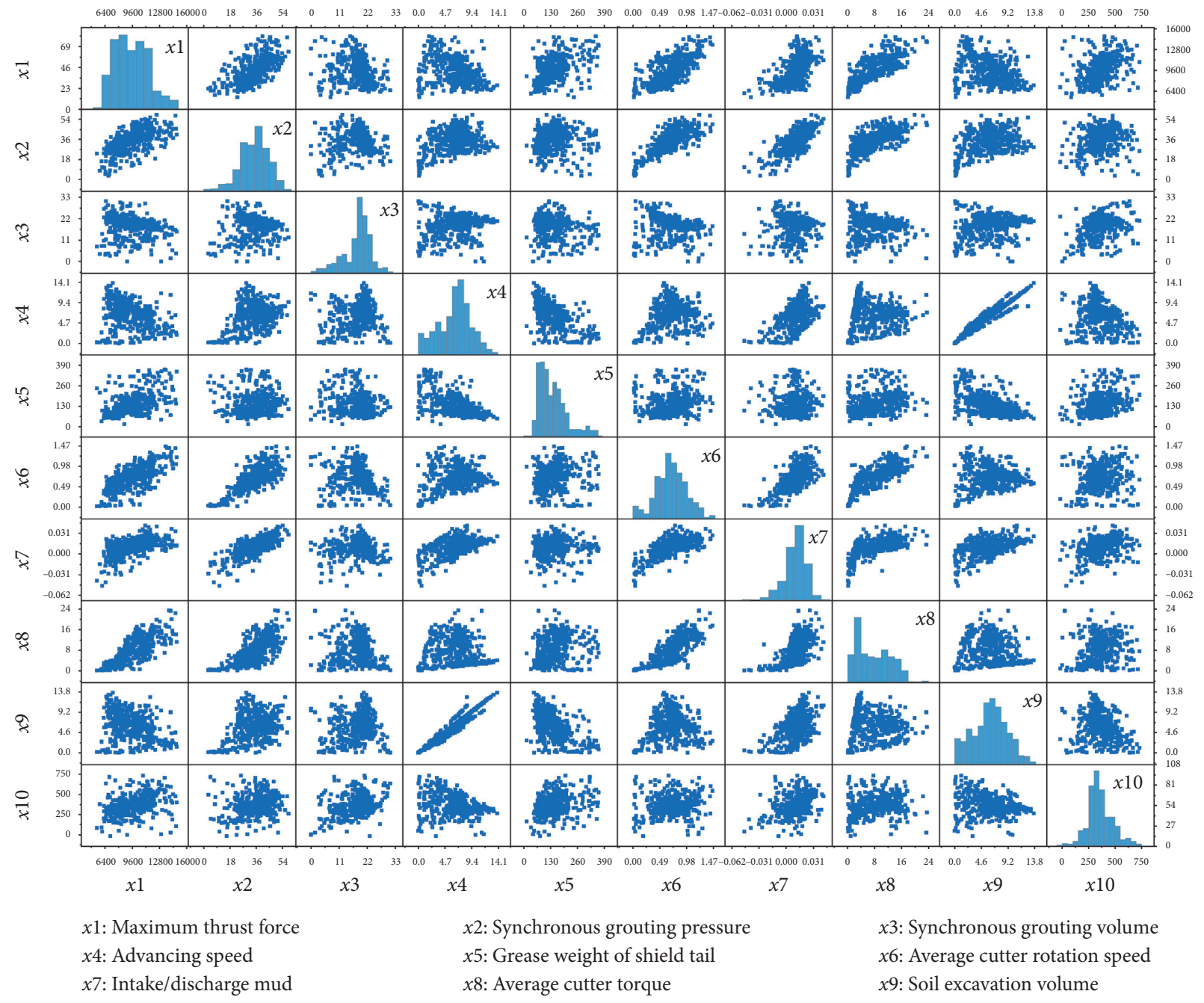

$x 10$ : Actual excavation volume

FIgURE 3: The scatter matrix for each input parameter.

4.3. Step 3: Performing GUA and GSA by PCE-eFAST Method. The eFAST is a variance decomposition method, which uses a periodic sampling method and a Fourier transformation to partition the whole variance of the model output and quantify the degree to which variation in each input factor accounts for the output variance [59]. A periodic sampling approach is used to generate a search curve in the parameter space, and partitioning is implemented by assigning the periodic sample of each parameter with a distinct frequency $[15,46]$. Then, a Fourier transformation is applied to the model output to measure how strongly a factor's frequency propagates from the input to the output, i.e., the variance contribution of the factor to the whole variance of the output [12].

First, in order to use the model evaluations more effectively, a conversion function in (8) is selected for the input parameters $x_{i}\left(i=1,2, \ldots, N_{t}\right)$.

$$
x_{i}(t)=\frac{1}{2}+\frac{1}{\pi} \arcsin \left[\sin \left(\lambda_{i} t\right)+v_{i}\right]
$$

where $v_{i}$ is a random phase in $[0,2 \pi]$. Here, $v_{i}=\pi / 3, \lambda_{i}$ represents the characteristic frequency of the input parameters, and $t$ is an independent variable for all input parameters. In $(-\pi, \pi), N_{t}=2 \omega \lambda_{\max }+1$ is taken as a uniform interval. $\omega$ is usually set to 4 or 6 , and $\lambda_{\max }$ is the maximum value of the sequence $\left\{\lambda_{i}\right\}$ that should satisfy the following nonlinear correlation: $\sum_{i=1}^{n} s_{i} \cdot \lambda_{i} \neq 0$. In the above formula, $s_{i}$ is an integer. Therefore, $y=f\left(x_{1}, x_{2}, \ldots, x_{10}\right)$ can be transformed into $y=f(t)$; that is,

$$
\begin{aligned}
f(t)= & f\left[X_{1}\left(\sin \lambda_{1}\right), X_{2}\left(\sin \lambda_{2}\right), \ldots,\right. \\
& \left.X_{10}\left(\sin \lambda_{10}\right)\right] t \in(-\pi, \pi) .
\end{aligned}
$$

The Fourier series expansion of $f(t)$ is

$$
y=f(t) \approx \sum_{-\left(N_{t}-1\right) / 2}^{\left(N_{t}-1\right) / 2}\left\{U_{j} \cdot \cos (j \cdot t)+V_{j} \cdot \sin (j \cdot t)\right\} .
$$

The Fourier coefficients $U_{j}$ and $V_{j}$ are defined as 


$$
\begin{aligned}
U_{j} & =\frac{1}{N_{t}} \sum_{k=1}^{N_{t}} f\left(t_{k}\right) \cdot \cos \left(j \cdot t_{k}\right), \\
V_{j} & =\frac{1}{N_{t}} \sum_{k=1}^{N_{t}} f\left(t_{k}\right) \cdot \sin \left(j \cdot t_{k}\right), \\
j \in Z & =\left\{-\frac{N_{t}-1}{2}, \ldots,-2,-1,0,1,2, \ldots,+\frac{N_{t}-1}{2}\right\}, \\
t_{k} & =\frac{\pi}{N_{t}}\left(2 k-N_{t}-1\right), k=1,2, \ldots, N_{t}, \\
z^{0} & =Z-\{0\}=\left\{-\frac{N_{t}-1}{2}, \ldots,-2,-1,1,2, \ldots,+\frac{N_{t}-1}{2}\right\} .
\end{aligned}
$$

The Fourier frequency spectrum curve is defined as $\Lambda_{p}=$ $U_{j}^{2}+V_{j}^{2}$ with $j \in Z, U_{j}, V_{j}, \Lambda_{p}$ having the following properties:

$$
\begin{aligned}
& U_{-j}=U_{j}, \\
& V_{-j}=V_{j}, \\
& \Lambda_{-j}=\Lambda_{j} .
\end{aligned}
$$

The variance of the model caused by the parameters' input changes can be expressed as the sum of the parameters $1,2,3, \ldots, \omega$ frequency doubling the spectral curves.

$$
W_{i}=\sum_{p \in Z^{0}} \Lambda_{p} \lambda_{i}=2 \cdot \sum_{p=1}^{M}\left(U_{p-\lambda_{i}}^{2}+V_{p-\lambda_{i}}^{2}\right)
$$

where $z^{0}=Z-\{0\}, \lambda_{i}$ is the integer frequency defined by the parameter $x_{i}$, and $P$ is the integer. Then, the total variance of the model is

$$
W=\sum_{p \in Z^{0}} \Lambda_{p}=2 \cdot \sum_{j=1}^{N_{t}-1} \Lambda_{j}
$$

The total variance of the model output $W$ and the variance of the model $W_{i}$ can be obtained by $U_{p}, V_{p}$, and $\lambda_{i}$. Since the total variance of the model output is obtained by parameters or coupling the parameters, the decomposition can be expressed as

$$
W=\sum_{i} W_{i}+\sum_{i \neq j} W_{i j}+\sum_{i \neq j \neq m} W_{i j m}+\cdots+W_{12, \ldots, k},
$$

where $W_{i j}$ is the variance (coupling variance) contributed by the input item $x_{i}$ through the input item $x_{j}$, and $W_{i j m}$ is the variance contributed by the input item $x_{i}$ through the error items $x_{j}$ and $x_{m} . W_{12}, \ldots, k$ is the variance contributed by the input item $x_{i}$ through the input items $x_{1}, x_{2}, \ldots, x_{k}$. Therefore, the first-order sensitivity index $R_{i}$ of the input item $x_{i}$ can be defined as (17) by the normalized processing:

$$
R_{i}=\frac{W_{i}}{W}
$$

The global sensitivity index reflects the sum of the input item, direct contribution rate, and interaction with input parameters indirectly to the variance of the model output. By decomposing the output variance of the model, the eFAST method can quantitatively obtain every order and global sensitivity of each error item. Therefore, the eFAST method can not only test the effect of the change in multiple error items on the input model results but also analyze the direct and indirect effects of the change of each input item on the simulation results.

The specific methods for calculating the total sensitivity are as follows. The input item $x_{i}$ was assigned to a larger frequency $\lambda_{i}$, and then, a set of smaller different integer frequencies was assigned to other input items. The selected integer frequency should meet the following relationship: $\lambda_{i} \geq 2 \omega \cdot \max \left\{\lambda_{i}^{\prime}\right\}$, so the frequency domain was divided into two parts: $\left[1, \omega \cdot \max \left\{\lambda_{i}^{\prime}\right\}\right]$ and $\left[\omega \cdot \max \left\{\lambda_{i}^{\prime}\right\}+1, N_{t}-1 / 2\right]$. Finally, the total sensitivity of the input item was calculated by

$$
\mathrm{ST}_{i}=\frac{\sum_{j=\omega \cdot \max \left\{\lambda_{i}^{\prime}\right\}}^{N_{t}-1 / 2}\left(U_{j}^{2}+V_{j}^{2}\right)}{\sum_{j=1}^{N_{t}-1 / 2}\left(U_{j}^{2}+V_{j}^{2}\right)}
$$

The aforementioned steps are summarized in Algorithm 1.

\section{The UA/SA for Superlarge Diameter Shield Excavation}

The data of this study comes from the Sanyang Road Highway-railway Tunnel (SRHT). The SRHT project of rail transit line 7 is located in Wuhan, China, with a total length of about $31.3 \mathrm{~km}$. This tunnel is designed for both road and subway use and constructed using two superlarge (diameters are 15.76 meters) mud-water balance shield machines. In the project of SRHT, especially in the compound stratum around the under-river section, the control of excavation stability is a vital problem. In the SRHT project, the BIMbased big data platform (BIM-BDP) has been established for the monitoring of construction parameters.

Global UA/SA aims to depict the entire set of possible model outcomes, together with their associated frequencies of occurrence [59]. We conducted SA for investigating the differences of outcome uncertainty derived from the variation in input parameters with eFAST. Besides, we identified influential input parameters by calculating both the MSI and the TSI. In line with $[59,60]$, for each output variable, a threshold of MSI $>0.01$ and TSI $>0.1$ was imposed to identify influential parameters.

5.1. Surrogate Model Validation. The surrogate modeling methods by gPCE described in Section 4.2 are used to construct the relationship between input and output parameters. Because the parameters in the BIM-BDP are nonlinear and constitute strongly coupled system, it is extremely difficult to use a finite number of sampling points to construct a very high accuracy surrogate model that can completely replace the dynamic model. Therefore, the adjusted error-squared [61], $R_{\text {adjusted }}^{2}$, is calculated to validate the accuracy of the surrogate models. They can be calculated by 


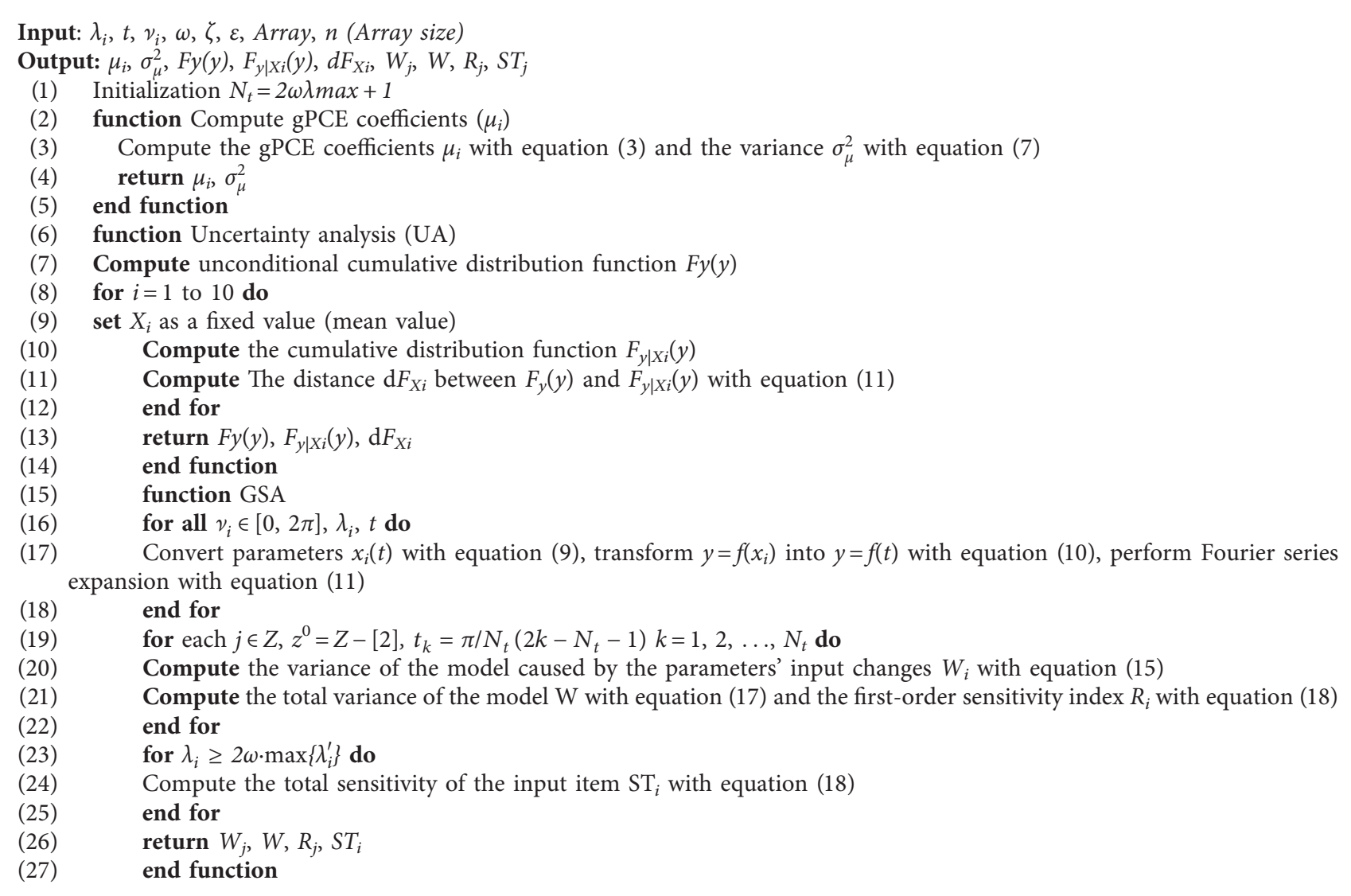

Algorithm 1: The eFAST-PCE algorithm for GUSA.

$$
\begin{aligned}
R_{\text {adjusted }}^{2} & =1-\left[\frac{n-1}{n-p-1}\right]\left(1-R^{2}\right), \\
R^{2} & =1-\frac{\sum_{i=n}^{n}\left(y_{i}-y_{i}^{*}\right)^{2}}{\sum_{i=1}^{n}\left(y_{i}-\bar{y}\right)^{2}}
\end{aligned}
$$

where $p$ is the total number of explanatory variables in the model (excluding the constant term), $n$ is the number of validation sample size, $y_{i}$ is the observed value, $y^{*}{ }_{i}$ is the value predicted by the surrogate model, and $\bar{y}$ is the average value of the observed value. Larger values of the $R_{\text {adjusted }}^{2}$ reveal that the surrogate model is a better approximation of the original dynamic model.

Figure 4 shows the scatters of the observation data in BIM-BDP and the prediction data by the surrogate model. Herein, the $R_{\text {adjusted }}^{2}$ values of $s 1$ and $s 2$ are 0.850 and 0.896 , respectively, using (18). Those values are all close to 1 , indicating that the surrogate model can predict the average magnitudes of $s 1$ and $s 2$ with high reliability. The black dash line in Figure 4 means that the observation data is equal to prediction data, and the red line is the fit line of the observation data versus prediction data. The small deviation of the above two lines also indicates the good fitting accuracy of the surrogate model.

\subsection{GSA Results}

5.2.1. The Impacts of Sample Size on the Sensitivity Measures. The goal of parameter GSA is to explore the entire input space with a reasonable sample size and to identify the sensitive parameters [62]. A key element of GSA is the sampling of input parameters for the simulation, and the sample size determines the computational cost of the analysis [46]. Different metrics of the train sample have been adopted to evaluate the accuracy of the surrogate model. The metamodel is built using a training set $(\mathbf{X} ; \mathbf{Y})$ of size $N_{\text {train }}$ that is much smaller than that of the total sample $N_{\text {total }}$, which is got from the BIM-BDP database. It is noteworthy that the values in the training set are continuously selected along the segment ring. Then, the prediction data by different metamodels can be validated with respect to the original observation data. In this research, 7 groups of training samples (sample sizes are $704,1408,2816,5632,11264,22528$, and 45056, respectively) are collected with the mean and standard deviation of the 10 input parameters by the Latin hypercube sampling (LHS) method. Then, the $s 1$ and $s 2$ can be obtained by running the surrogate model. The evolution of the MSI and TSI with increasing sample size is shown in Figure 5. 


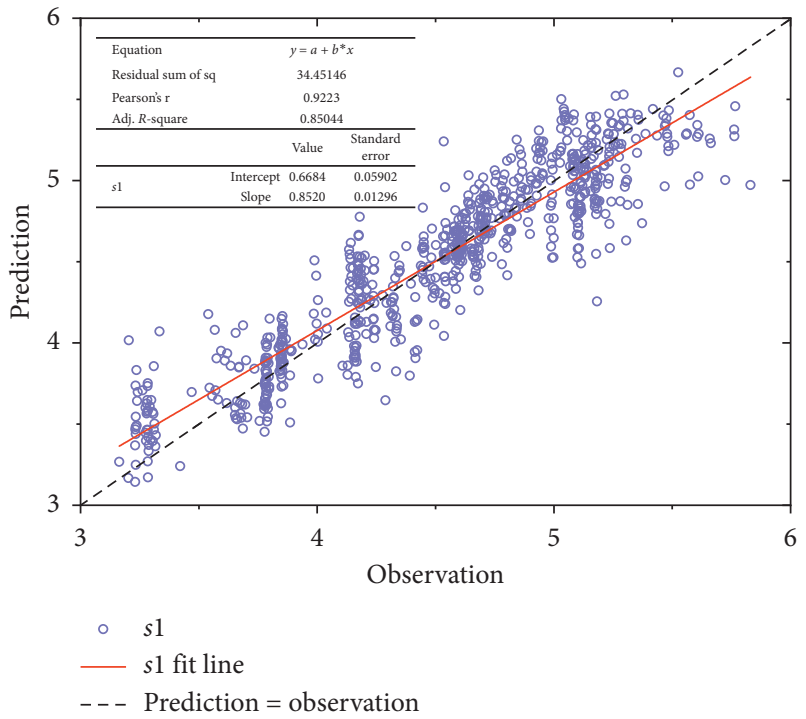

(a)

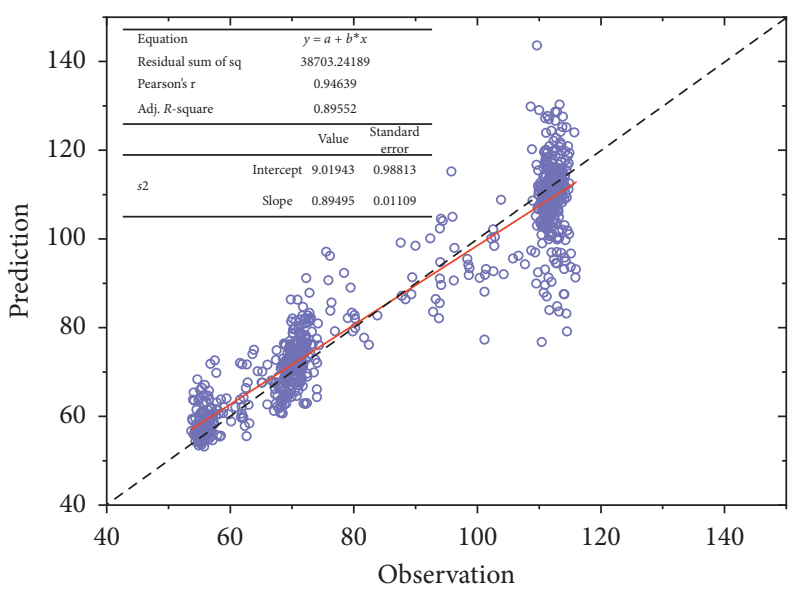

- $s 2$

— $s 2$ fit line

- - - Prediction $=$ observation

(b)

FIGURE 4: Scatter plot of observations and surrogate model enabled predictions for different outputs of output parameters: (a) bubble chamber pressure ( 1 ); (b) deviation angle (s2).

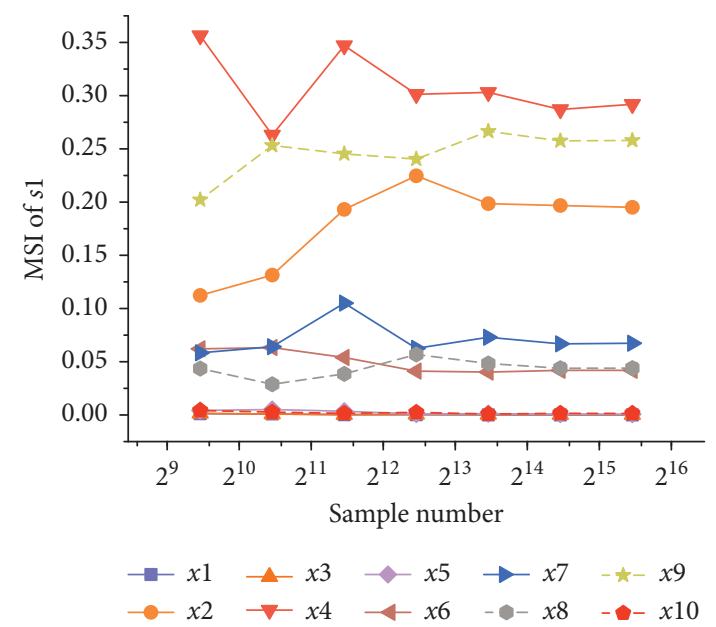

(a)

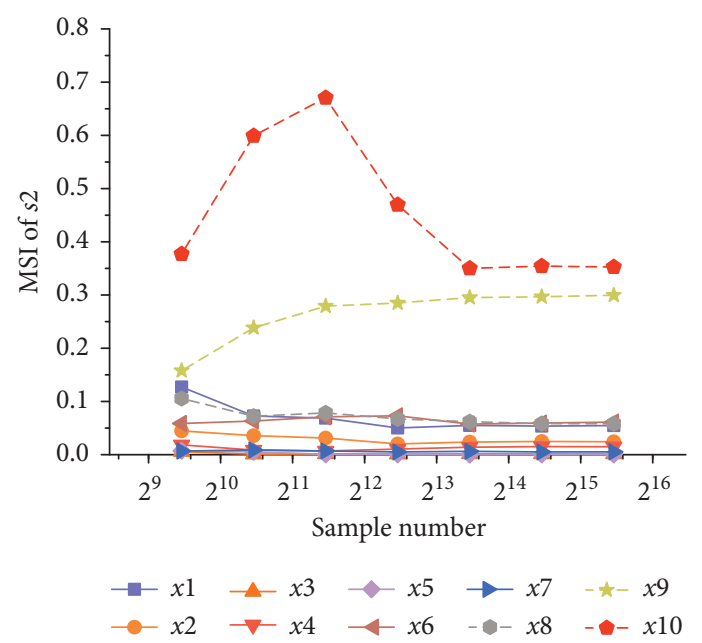

(c)

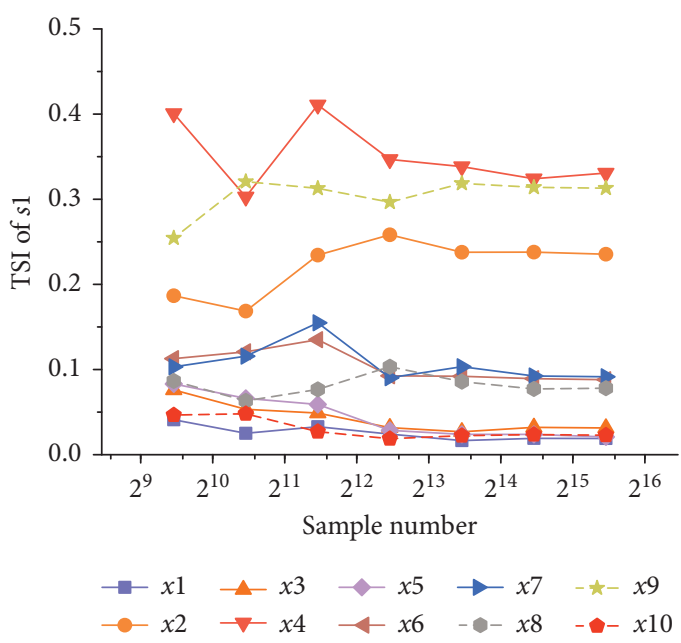

(b)

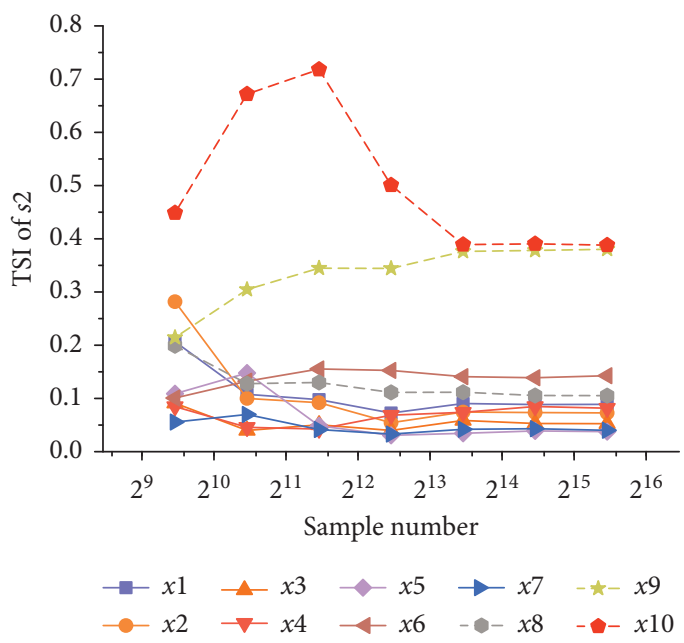

(d)

Figure 5: Parameter sensitivity rankings of the eFAST method for (a) MSI of $s 1$, (b) TSI of $s 1$, (c) MSI of $s 2$, and (d) TSI of $s 2$. 
With regard to bubble chamber pressure (s1), the MSIs and TSIs of input parameters vary greatly when the sample size is less than 22528. The most important parameter, advancing speed ( $x 4)$, is chosen as an example to exhibit the results of SA. The MSIs of advancing speed are 0.356, 0.263, 0.347, 0.301, $0.303,0.287$, and 0.291 , respectively, with the sample size increasing from 704 to 45056; meanwhile, the TSIs are 0.401, $0.303,0.411,0.347,0.338,0.324$, and 0.331 , respectively. Compared with the MSI and TSI, the input parameter rankings are almost the same, only if the sample value is 1408 .

With regard to deviation angle (s2), the MSIs and TSIs of input parameters vary greatly when the sample size is less than 11264. The most important parameter is actual volume excavation ( $x 10)$, which is chosen as an example, and the MSIs of actual volume excavation are 0.377, 0.599, 0.671, $0.470,0.350,0.354$, and 0.353 , respectively, with the sample size increasing from 704 to 45056; meanwhile, the TSIs are $0.449,0.672,0.718,0.501,0.389,0.391$, and 0.388 , respectively. Compared with the MSI and TSI, the input parameter rankings are the same. Therefore, if the objective of the parameter GSA is solely to calculate an input parameter ranking, then the eFAST analysis can be applied with a sample size of 22528 to yield a reliable ranking result.

From Figure 5, the sample size is the main determining factor for the convergence of the MSI and TSI with the surrogate model. The sample size over 22528 yields the most stable sensitivity indices. When the sample size is small, i.e., only 1408, the MSI of advancing speed ( $x 4$ ) shows strong variations and cannot reach a stable convergence result. For most of parameters, fewer than 11264 samples are not sufficient to reach a stable value. In general, it appears from the plots that a sample size of greater than 22528 is required to reach the final converged value for the most of parameters.

For the input parameter with the highest SIs, such as advancing speed $(x 4)$ to $s 1$ or actual volume excavation ( $x 10)$ to $s 2$, the final stable sensitivity index value is attained rather slowly, and greater fluctuations are observed. Convergence can be obtained for this type of parameter under the condition of a large sample size. However, those insensitive parameters are more prone to minor fluctuations that can appear with increasing sample size. For those insensitive parameters, the SA can quickly obtain convergence. These results are consistent with the research of [53], which verifies the robustness and universality of this study.

5.2.2. The MSIs and TSIs of the Entire Section. Figure 6 reports the full results about MSIs and TSIs for all 10 input parameters on the two output parameters, under the sample size of 22528 (according to the conclusion in Section 5.2.1). The influential parameters (corresponding to MSI $>0.01$ and TSI $>0.1$ ) are found for two outputs.

For the output bubble chamber pressure (s1), the synchronous grouting pressure ( $x 2)$, advancing speed ( $x 4)$, cutter rotation speed ( $x 6)$, ratio of intake/discharge mud ( $x 7)$, cutter torque $(x 8)$, and soil excavation volume $(x 9)$ are influential parameters according to the MSIs, whereas synchronous grouting pressure ( $x 2)$, advancing speed ( $x 4)$, and soil excavation volume $(x 9)$ are influential parameters according to the TSIs. The most important parameter is advancing speed ( $x 4)$ reaching an MSI of up to 0.292 and a TSI of up to 0.331 . The next important parameter, soil excavation volume $(x 9)$, reaches an MSI value of up to 0.258 and a TSI value of up to 0.313 . The third important parameter, synchronous grouting pressure ( $x 2)$, reaches an MSI value of up to 0.195 and a TSI value of up to 0.235 . Besides, the MSIs and TSIs of other input parameters are much lower than the above-mentioned three parameters.

For the output deviation angle (s2), the maximum thrust force $(x 1)$, synchronous grouting pressure ( $x 2)$, advancing speed $(x 4)$, cutter rotation speed $(x 6)$, cutter torque $(x 8)$, soil excavation volume $(x 9)$, and actual volume excavation ( $x 10)$ are influential parameters according to the MSIs, while cutter rotation speed $(x 6)$, cutter torque $(x 8)$, soil excavation volume $(x 9)$, and actual volume excavation $(x 10)$ are influential parameters according to the TSIs. The most important parameter is actual volume excavation $(x 10)$ reaching an MSI of up to 0.353 and a TSI of up to 0.388 . The next important parameter, soil excavation volume $(x 9)$, reaches an MSI value of up to 0.299 and a TSI value of up to 0.380. The MSIs and TSIs of other input parameters are much lower than the above two parameters.

Comparing the MSIs and TSIs in Figure 6, we find that the impact of an input parameter's interaction with other parameters on the outputs (TSI-MSI) can be much greater than that of the parameter's own contribution (MSI); for example, the MSI and TSI of actual volume excavation ( $x 10)$ to bubble chamber pressure (s1) are 0.002 and 0.024 , where the TSI is ten times the MSI. In this study, the TSI, which includes the effects of parameter interactions, yields basically the same ranking of the parameters as the MSI (as in Figure 6). Parameter importance may be better determined by the TSI, which is likely to give a better quantitative view of the overall parameter importance over the whole simulation parameter than indices computed for the individual parameter by MSI.

5.3. UA Results. We use global UA to study the propagation of uncertainty from basic input parameters to output parameters, and to quantify the confidence of the model output from existing data and parameter estimates. In this study, we characterize the well-trained metamodel by varying all of the input factors of the metamodel within a certain range. This process can be divided into four steps: Step 1, defining the type of random distribution, including Beta, Logistic, and Laplace in the current study; Step 2, creating a random sample of the input based on the selected distribution and introducing a random error into the model input; Step 3, running the model using random samples as input; Step 4, obtaining the error of each parameter to obtain a 95\% confidence interval.

As mentioned above, the input parameters have their initial conditions and a specific area. Next, the LHS method is used to generate inputs based on the distribution, and then the output is generated using the PCE-eFAST model. After 


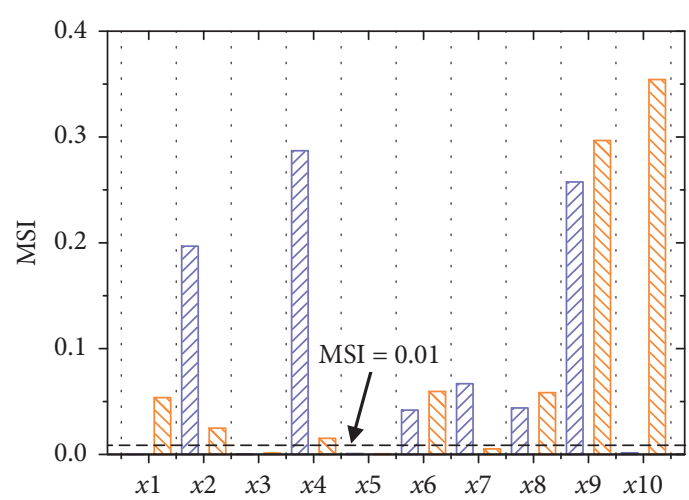

ZIIA $s 1$

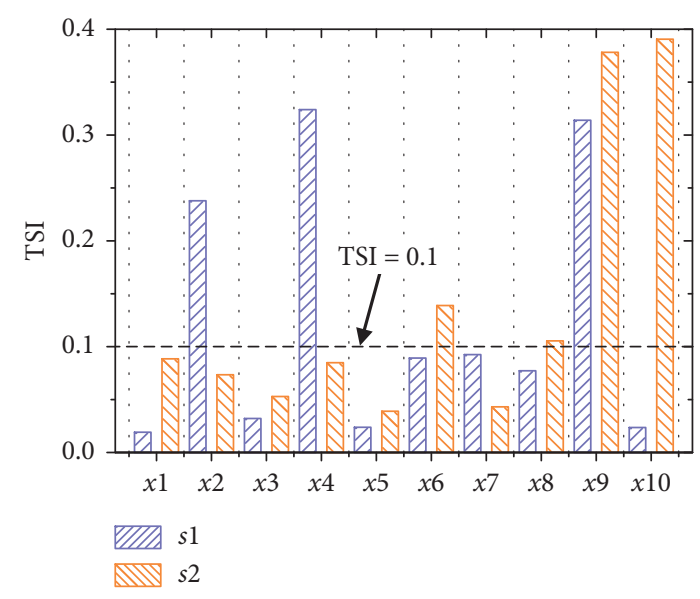

(b)

FIgURE 6: The full results of MSIs and TSIs for all 10 input parameters on the two output parameters, under the sample size of 22528: (a) MSI; (b) TSI.

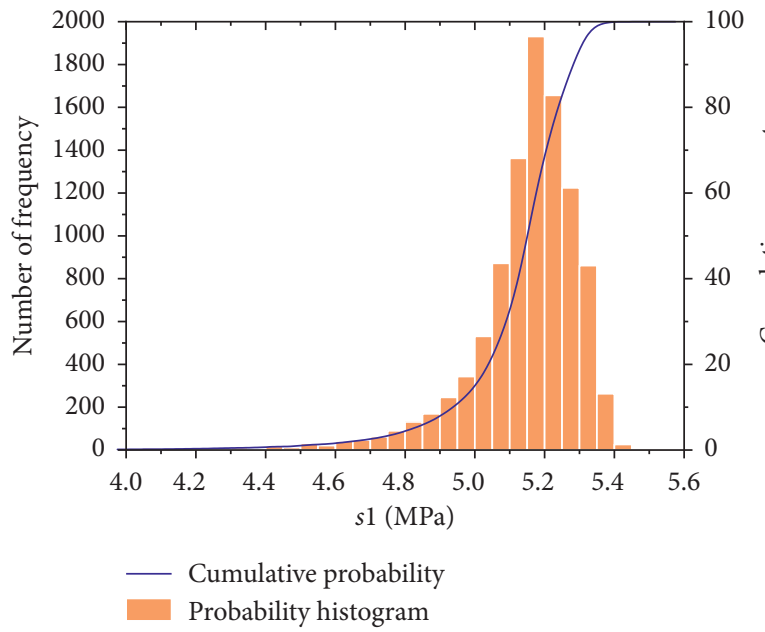

(a)

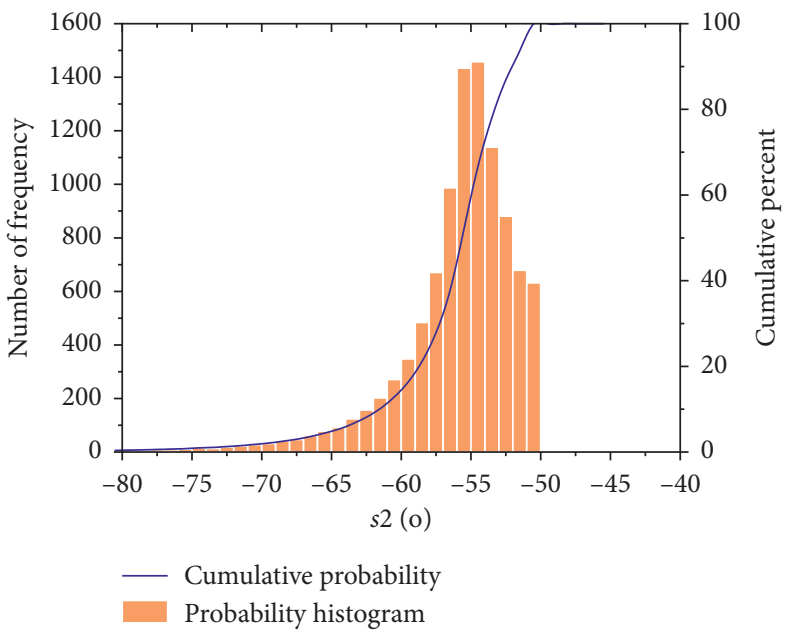

(b)

Figure 7: Results of global UA of shield machine intrinsic parameters within different model outputs in the baseline scenario: (a) average bubble chamber pressure per ring (s1); (b) deviation angle per ring $(s 2)$.

several iterations, the uncertainty of the PCE-eFAST model will be represented by CDF of the model outputs.

Figure 7 shows the results of the UA of the inherent parameters of the shield machine in different model outputs. Due to the uncertainty of the input parameters and their interaction, the model output $s 1$ (average bubble chamber pressure per ring) ranges from 4.4 to $5.4 \mathrm{MPa} ; 2$ (deviation angle per ring) ranges from $-80^{\circ}$ to $-50^{\circ}$.

This study takes $s 1$ (average bubble chamber pressure per ring) as an example. Figure 8 shows the comparison between the univariate uncertainty $\mathrm{CDF}$ curve and the baseline. Taking Figure 8(a) as an example, set $x 1$ as the average of its distribution, the blue thick solid line is the baseline CDF of the model output $s 1$ (the same as that shown in Figure 7(a)), and the red dotted line is the univariate of the model output $s 1$ uncertainty CDF. If the two curves are highly similar, this indicates that the change in value $x 1$ has no significant effect on the model result $s 1$. Obviously, the factors $x 2$ (Figure 8(b)), $x 4$ (Figure 8(d)), and $x 9$ (Figure 8(i)) have the greatest impact on model output because their univariate uncertainty CDF curves are very different from baseline CDF. At the same time, the factors $x 1$ (Figure $8(\mathrm{a})$ ), $x 2$ (Figure $8(\mathrm{c})$ ), $x 3$ (Figure $8(\mathrm{e})$ ), and $x 4$ (Figure $8(\mathrm{j})$ ) have little effect on the model output because their univariate uncertainty CDF curves almost coincide with the baseline. The results of the UA are identical with those obtained in Figure 6, which indicates that the method can identify inputs that have a significant impact on the output. In this way, the UA result of the proxy model method can be verified in some aspects. 

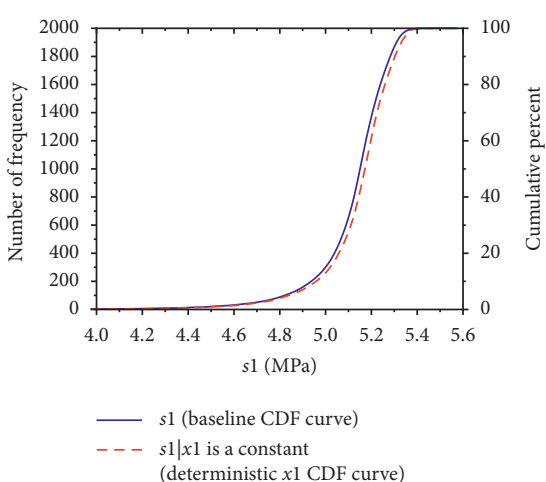

(a)

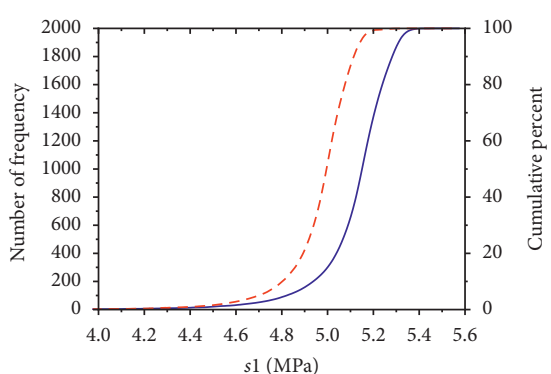

$\begin{array}{ll}- & s 1 \text { (baseline CDF curve) } \\ --- & s 1 \mid x 4 \text { is a constant } \\ & \text { (deterministic } x 4 \text { CDF curve) }\end{array}$

(d)

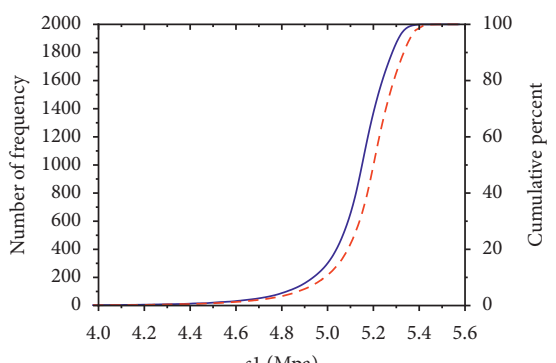

$s 1$ (Mpa)

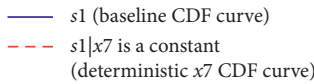

(g)

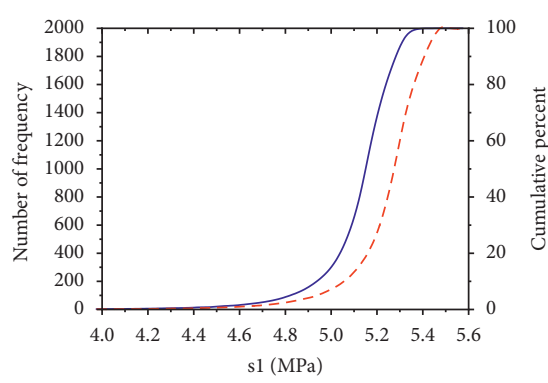

$\begin{array}{ll}- & s 1 \text { (baseline CDF curve) } \\ --- & s 1 \mid x 2 \text { is a constant } \\ & \text { (deterministic } x 2 \text { CDF curve) }\end{array}$

(b)

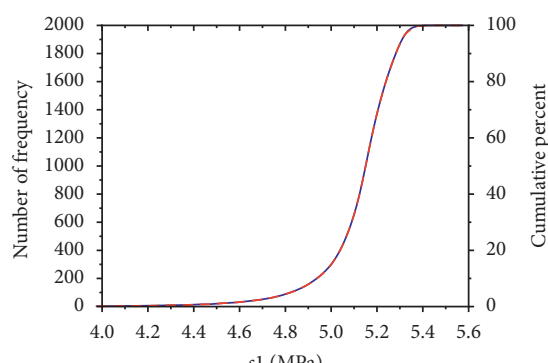

$s 1(\mathrm{MPa})$

_ s1 (baseline CDF curve)

- - $s 1 \mid x 5$ is a constant

(deterministic $x 5$ CDF curve)

(e)

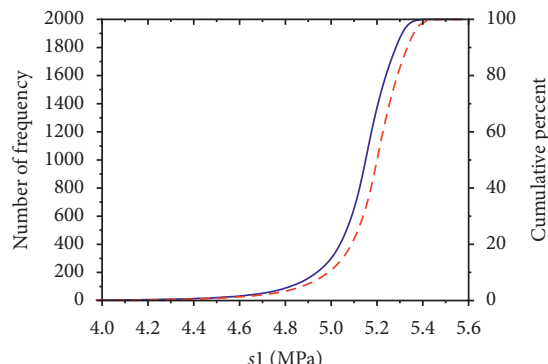

_ $s 1$ (baseline CDF curve)

- - - $s 1 \mid x 8$ is a constant

(deterministic $x 8 \mathrm{CDF}$ curve)

(h)

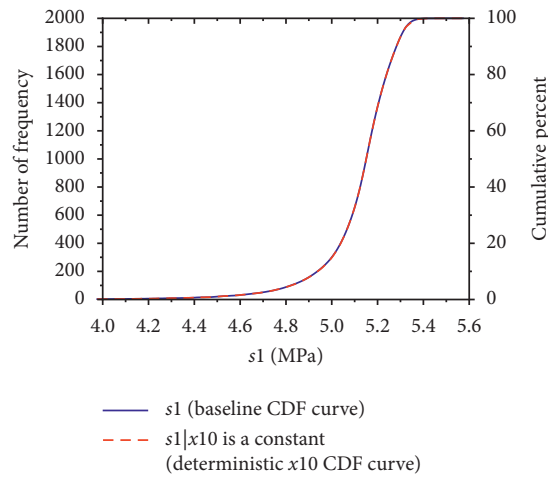

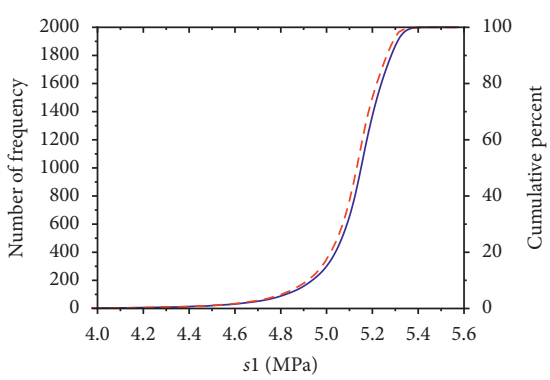

$s 1$ (baseline CDF curve)

$s 1 \mid x 3$ is a constant

(deterministic $x 3 \mathrm{CDF}$ curve)

(c)

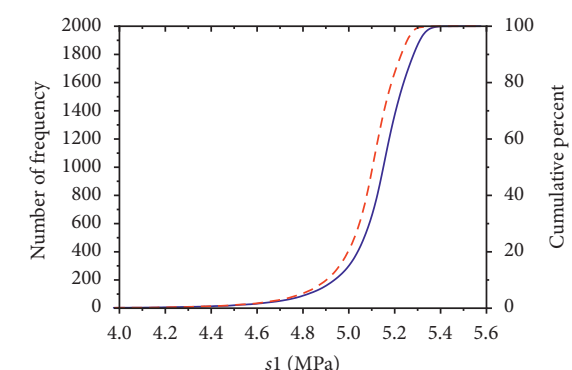

__ $s 1$ (baseline CDF curve)

_. $s 1 \mid x 6$ is a constant

(deterministic $x 6$ CDF curve)

(f)

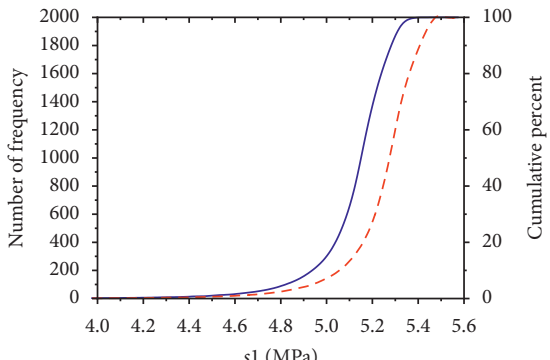

_ $s 1$ (baseline CDF curve)

- - $s 1 \mid x 9$ is a constant

(deterministic $x 9$ CDF curve)

(i)

(j)

FIGURE 8: Comparison of single-deterministic-parameter CDF curves and baseline CDF curves for the model output s1 (average bubble chamber pressure per ring). 


\section{Discussion}

The BIM-BDP is used for monitoring shield construction parameters and status during tunnel construction. The database of BIM-BDP, including BIM database, real-time collection database, and manual filling database, supports shield tunneling management and further analysis. Then, we performed GSA of the control parameters of the shield machine from BIM-BDP data on SRHT, under high uncertainty. We use the eFAST which has proven to be among the most reliable and efficient GSA methods $[13,59]$. The finding that significant differences exist in parameters identified as influential and noninfluential, in parameter importance rankings, and in the magnitude of MSI and TSI under high uncertainty has not been reported previously. Throughout our research, it is found that the set of significant first-order MSI and total-order TSI sensitivity indices returned by eFAST exhibit the same ranking.

According to the previous research [63, 64], LHS develops a procedure to establish correlations between sampled values; hence, the LHS has been adopted as a sampling method in contrast to the samples by the eFAST method. Furthermore, the Pearson correlation coefficient (PCC) (see [65]) has been used to investigate the correlation between samples. The PCCs of samples from BIM-BDP data, LHS, and eFAST are used to obtain 1000 random parameter combinations, and the LHS and eFAST method are illuminated in Figure 9. From Figure 9, the sample PCC from BIM-BDP data is similar to that from LHS. However, the sample PCC from the eFAST method is almost close to zero, which means the eFAST method cannot fit correlation coefficients of the input samples effectively. To be specific, the maximum correlation coefficient from BIM-BDP data is between $x 4$ and $x 9$, which is 0.938 and indicates a strong relevance between $x 4$ and $x 9$. In contrast, the maximum correlation coefficient of $x 4$ and $x 9$ from LHS is 0.947 , which is very similar to BIM-BDP data. However, this coefficient of $x 4$ and $x 9$ from the eFAST method is 0.001 , which is almost close to zero and quite different from the coefficient from BIM-BDP data and LHS.

In order to elucidate the sample technology by the eFAST method, the parallel coordinates plot of selected samples is shown in Figure 10, which reveals the distribution of samples within their variability ranges. In Figure 10, the parameter ranges are standardized to percentiles and allowed for comparison across parameters. Figures 10(a) and 10(b) are the samples from the BIM-BDP system, Figures $10(\mathrm{c})$ and $10(\mathrm{~d})$ are the samples from LHS, and
Figures 10(e) and 10(f) are the samples from the eFAST method. Figures 10(a), 10(c), and 10(e) show that the samples vary from the entire range, while Figures $10(\mathrm{~b})$, $10(\mathrm{~d})$, and $10(\mathrm{f})$ indicate all the samples with the $x 4$ varying from 0.2 to 0.3 (set as an example to illustrate the correlation coefficient of $x 4$ to other parameters). Comparing Figures 10(a), 10(c), and 10(e), we find that the samples do not fill the entire ranges of the parameters from BIM-BDP system in Figure 10(a), while from LHS and eFAST method, the samples are set as the desired distribution (e.g., uniform and normal) over the entire range in Figures 10(b) and 10(c).

The samples with the $x 4$ varying from 0.2 to 0.3 are extracted from the entire range. Figure 9 shows that relevance between $x 4$ and $x 9$ is very intense, which can be also demonstrated by Figure 10. From the BIM-DMSCC system, the sample range of $x 4$ is very similar to $x 9$; to be specific, with the $x 4$ varying from 0.2 to 0.3 , the $x 9$ almost varied from 0.18 to 0.41 in Figure 10(b). For LHS, the sample range of $x 4$ is also very similar to $x 9$, and with the $x 4$ varying from 0.2 to 0.3 , the $x 9$ almost varies from 0.16 to 0.41 in Figure 10(d). However, as to the eFAST method, with the $x 4$ varying from 0.2 to 0.3 , the $x 9$ almost varies from 0.01 to 0.81 in Figure 10(f). This is because the sampling procedure implemented in eFAST defines a sinusoidal function of a particular frequency for each input parameter [65], i.e., the search curve in equation (8), that assigns a value to $x$ based on the sample number 1 through the total number of samples per search curve, $N_{t}$. The choice of the sinusoidal function depends on the distribution of parameter values desired (e.g., uniform, normal). Hence, the correlation coefficients of the input samples by the eFAST method are quite different from the correlation coefficients of the inputs from BIM-BDP data.

In general, the computational execution time of the model is the major concern when performing the eFAST method [65]. However, the sampling procedure of the eFAST method can be further investigated for the influence on SA results. Hence, more SA methods need to be adopted for comparisons, such as Morris elementary effects [66, 67] and Sobol's indices [68, 69]. In addition, only 10 input parameters and two output parameters have been regarded as significant parameters and investigated in this study; however, the control system of the superlarge diameter shield machine is very complicated and has more than 900 groups of control parameters. This is because it is difficult to use the metamodel to fit the relevance of so many parameters. More effort to expand the input-output index system would further improve the application scope of GSA results. 


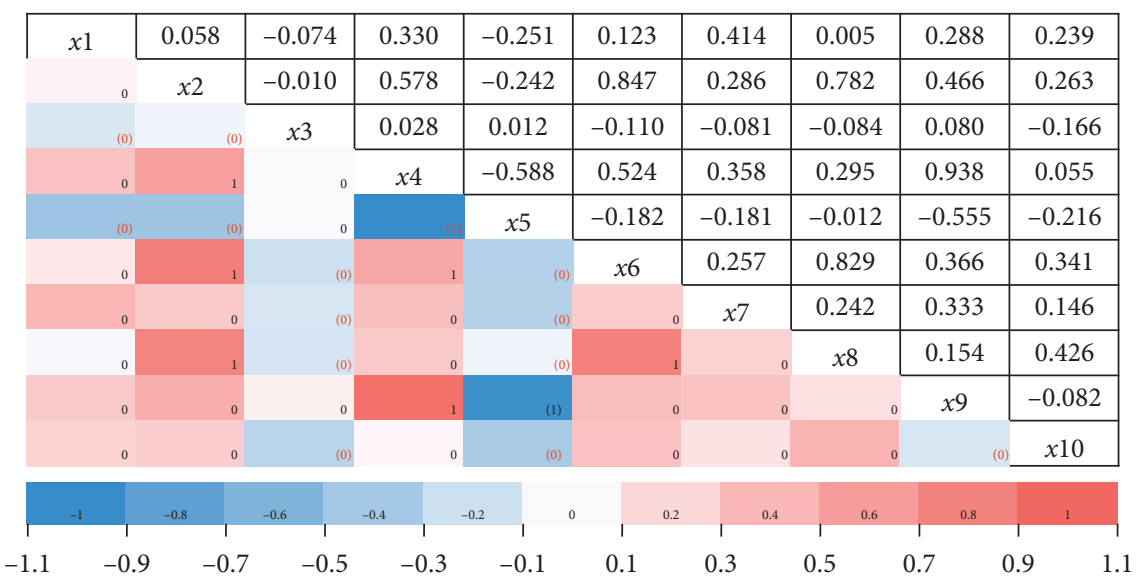

(a)

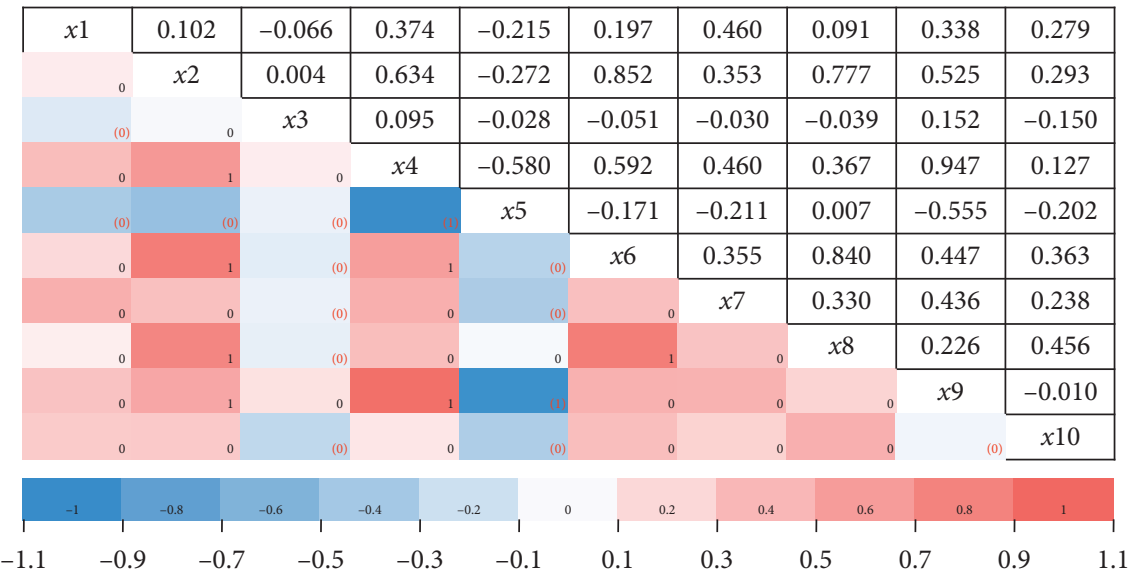

(b)

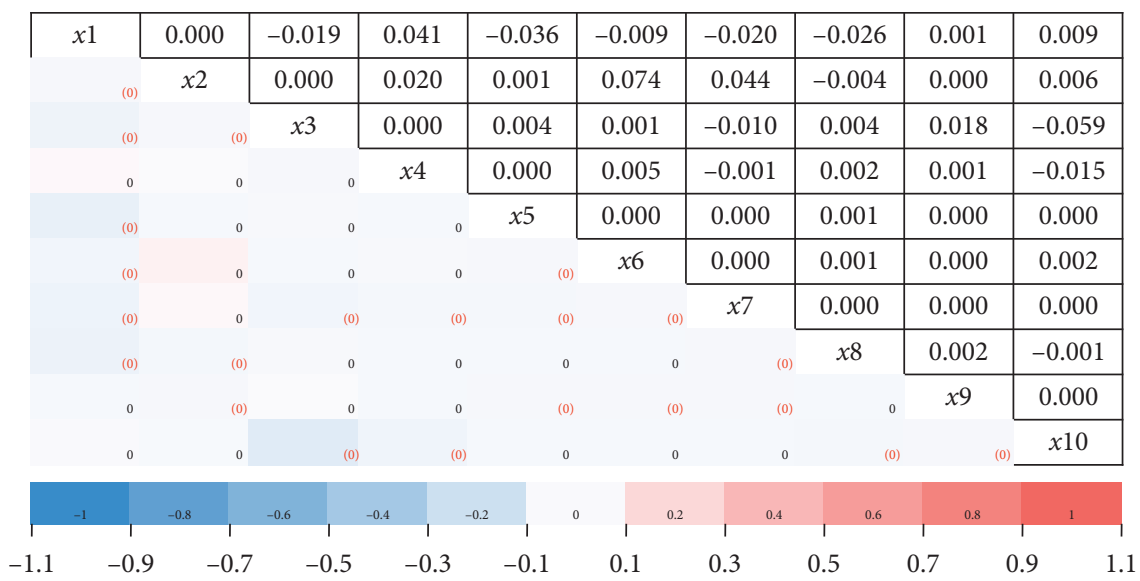

(c)

Figure 9: The PCCs of samples from BIM-BDP data, LHS, and eFAST method. Colors of the grid cells in the lower-left corner represent the PCCs, and numbers in the upper right represent their specific values: (a) PCCs of samples from BIM-DMSCC system; (b) PCCs of samples from LHS; (c) PCCs of samples from eFAST method. 


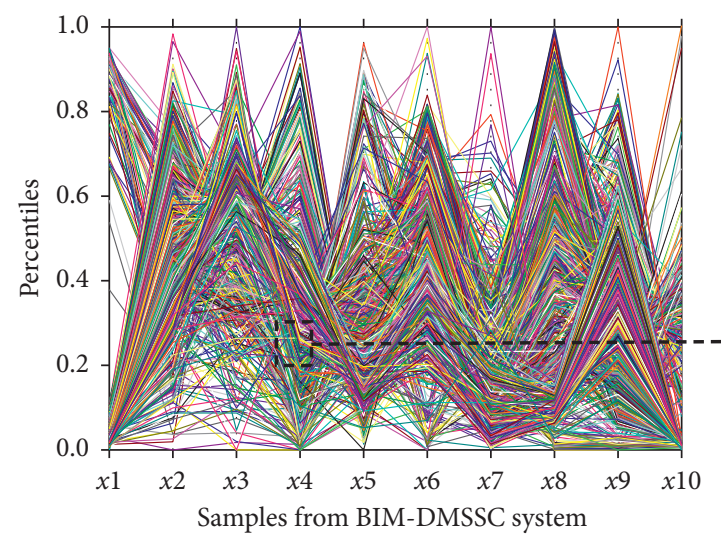

(a)

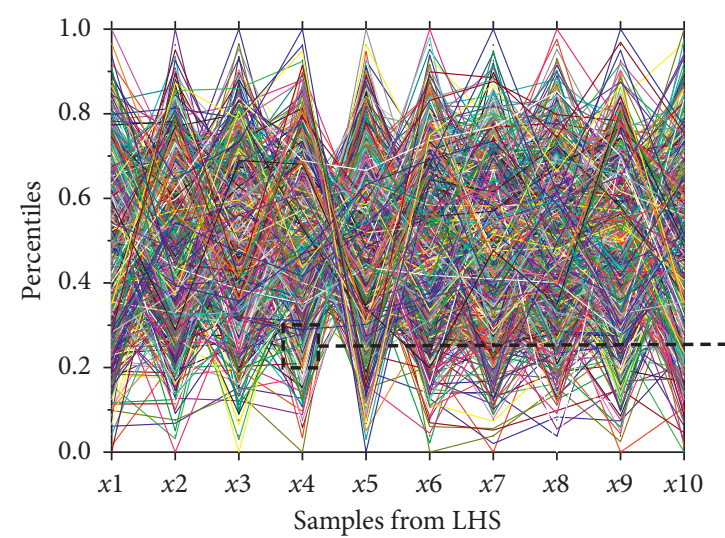

(c)

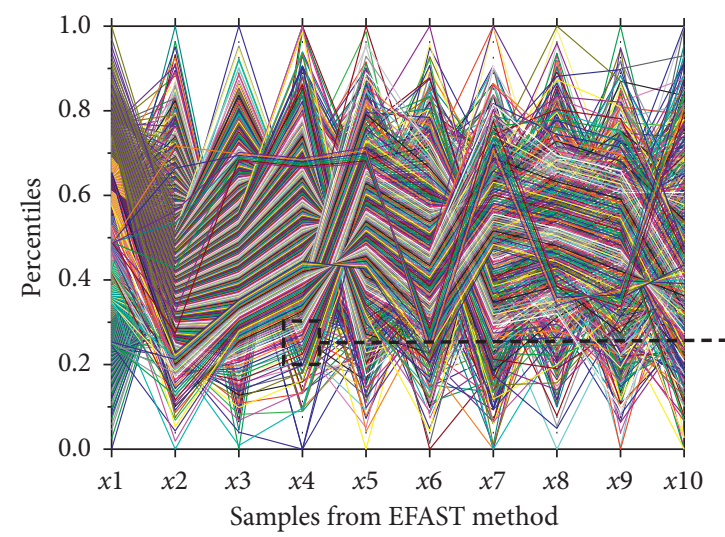

(e)

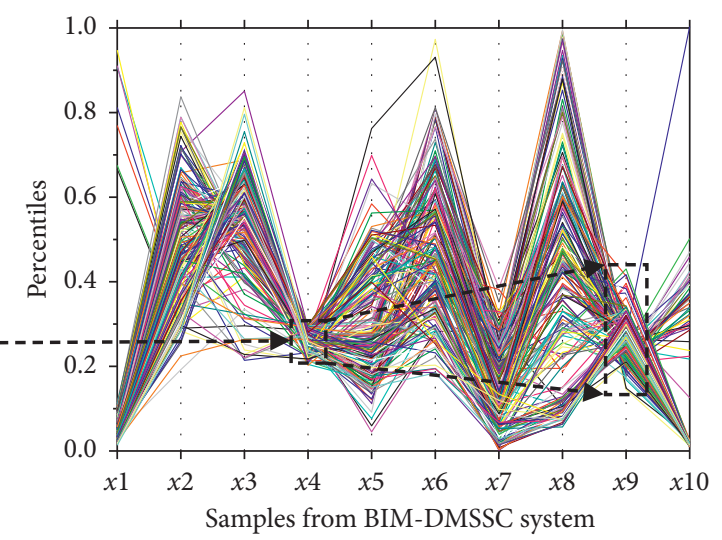

(b)

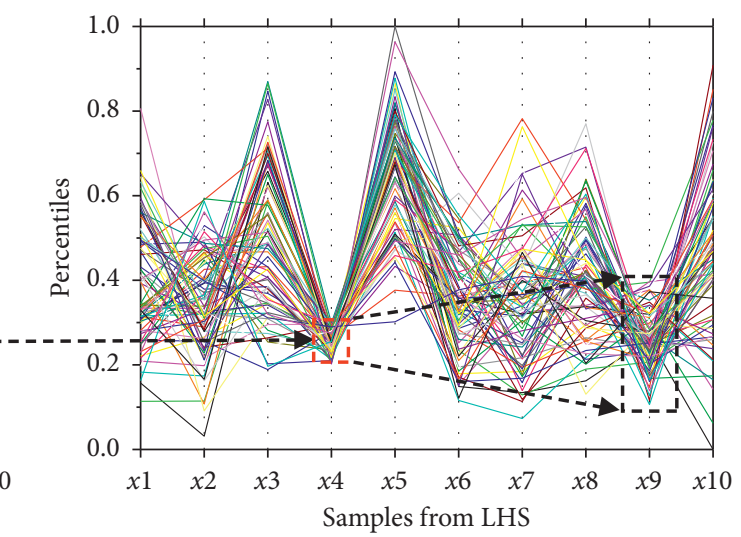

(d)

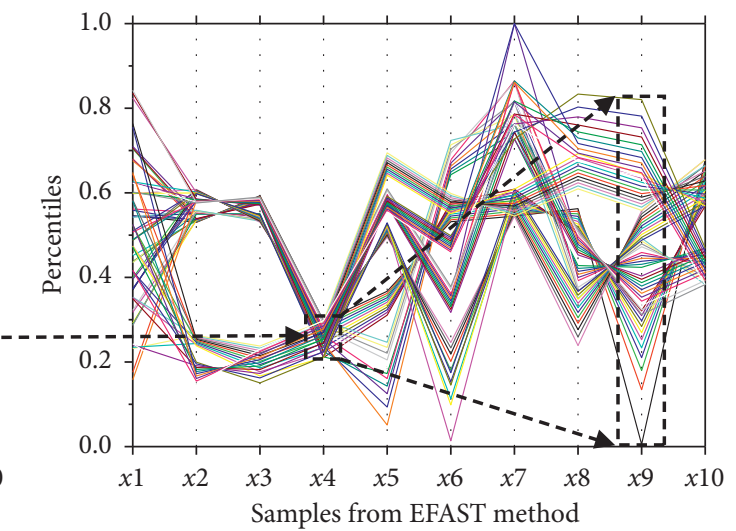

(f)

Figure 10: Parallel coordinates plot of samples from BIM-BDP data, LHS, and eFAST method: (a) samples from BIM-BDP; (b) samples from BIM-BDP with $x 4$ varying from 0.2 to 0.3 ; (c) samples from LHS; (d) samples from LHS with $x 4$ varying from 0.2 to 0.3 ; (e) samples from eFAST method; (f) samples from eFAST method with $x 4$ varying from 0.2 to 0.3 . If parameter lines of $x 4$ varying from 0.2 to 0.3 cover the entire range of a parameter, for instance, lines on $x 9$ in (f), the correlation coefficient is low. If they concentrate in a subrange, for instance, $x 9$ in (b) and (d), the correlation coefficient is high. 


\section{Conclusion}

It is important to control and adjust the shield parameters for excavation stability, especially in the SRHT project, which needs to cross 1365 meters of soft-hard composite strata in the under-river section. In this paper, BIM technology has been utilized to manage the essential project data of SRHT project in digital format throughout shield excavation, and then the GSA framework has been adopted to investigate the correlation between parameters from the BIM-BDP, which can help the administrators optimize their management scheme of shield parameters during the tunnel excavation.

SA can help the administrator identify the importance of the parameters, which can reduce the epistemic uncertainty of project safety during tunnel excavating. In addition, SA helps the administrator determine what type of additional information of parameters should be gathered to enhance project safety and reliability. For the output $s 1$, the $x 2, x 4, x 6, x 7, x 8, x 9$ are influential parameters according to the MSIs, while $x 2, x 4$, and $x 9$ are influential parameters according to the TSIs. The most important parameter is $x 4$ reaching an MSI of up to 0.292 and a TSI of up to 0.331 . The next important parameter, $x 9$, reaches an MSI value of up to 0.258 and a TSI value of up to 0.313 . The third important parameter, $x 2$, reaches an MSI value of up to 0.195 and a TSI value of up to 0.235 . Besides, the MSIs and TSIs of other input parameters are much lower than the above three parameters. For the output $s 2$, the $x 1, x 2, x 4, x 6, x 8, x 9, x 10$ are influential parameters according to the MSIs, whereas $x 6, x 8, x 9$, $x 10$ are influential parameters according to the TSIs. The most important parameter is $x 10$ reaching an MSI of up to 0.353 and a TSI of up to 0.388 . The next important parameter, $x 9$, reaches an MSI value of up to 0.299 and a TSI value of up to 0.380 . Besides, the MSIs and TSIs of other input parameters are much lower than those of the above two parameters.

The GSA from the BIM-BDP can determine the quantitative correlation between shield parameters. The decision maker can then evaluate the parameter adjustment strategy during shield advancing. These findings are of general value and can be extrapolated to other similar projects under high uncertainty. Some subsequent research can be investigated in the future. Only ten input parameters were considered in this study. However, it is necessary to study more shield machine control parameters to promote the establishment of more effective models and achieve specific goals more effectively. More complex and effective hybrid models can be studied to more accurately predict the stability of the excavation and optimize the construction plan. In addition, with the accumulation of more data, the modeling accuracy will be greatly improved. Future work can focus on how to reduce system uncertainty by the GSA technology and how to enlarge the application scope. This is expected to reduce system uncertainty and expand its application range through GSA technology.

\section{Nomenclature}

$\Omega: \quad$ The sample space

F: $\quad$ The $\sigma$-algebra collection of all measurable events belonging to $\Omega$
P: $\quad$ A probability measure indicating the likelihood of occurrence of each event

Z: $\quad$ A set of uncorrelated random variables that represent the uncertainty in the system

$\mu_{i}: \quad$ The gPCE coefficient

E: $\quad$ Expectation

$\Psi_{i}$ : Wiener-Hermite polynomial chaos

N: $\quad$ Positive integer

P: The probability distribution density

E: Weights

P: $\quad$ Polynomial order

$\|\cdot\|: \quad$ Euclidean distance

$\sigma_{\mu}^{2}$ : $\quad$ Variance

$x_{i}: \quad$ The input parameters

$\lambda_{i}$ : The characteristic frequency of the input parameters

$T: \quad$ Independent variable for all input parameters

$v_{i}: \quad$ A random phase between $[0,2 \pi]$

Q: $\quad 4$ or 6

$\Lambda_{\text {max }}:$ The maximum value of the sequence $\left\{\lambda_{i}\right\}$

$s_{i}: \quad$ Integer

$U_{j}, V_{j}:$ Fourier coefficients

$\Lambda p: \quad$ Fourier frequency spectrum curve

$W_{i}$ : The variance of the model caused by the parameters' input changes

$W: \quad$ The total variance of the model

Ri: $\quad$ The first-order sensitivity index

ST $i$ : The total sensitivity of the input item

$R^{2}$ : $\quad$ Coefficient of determination

$P$ : $\quad$ The total number of explanatory variables in the model

$N$ : $\quad$ The number of validation sample size

$y_{i}: \quad$ The observed value

$y_{i}{ }_{i}: \quad$ The value predicted by the surrogate model

$\bar{y}$ : $\quad$ The average value of the observed value

$R: \quad$ The set of real numbers

UA/ Uncertainty and sensitivity analysis

SA:

TBM: Tunnel boring machine

BIM: Building Information Modeling

SRHT: Sanyang Road Highway-Railway Tunnel

GSA: Global sensitivity analysis

MSI: Main sensitivity index

TSI: Total sensitivity index

gPCE: Generalized polynomial chaos expansion

eFAST: Extended Fourier amplitude sensitivity test

PDF: Probability distribution functions

CDF: Cumulative distribution functions

PCC: Pearson correlation coefficient

LHS: Latin hypercube sampling.

\section{Data Availability}

The data generated by the superlarge tunnel boring machine used to support the findings of this study have not been made available because of the nondisclosure agreement the research team signed with the property owner and the contractor. 


\section{Conflicts of Interest}

The authors declare that they have no conflicts of interest.

\section{Acknowledgments}

The study was supported by the National Natural Science Foundation of China (no. 71732001) and the Fundamental Research Funds for the Central Universities of China (Grant no. 2018KFYYXJJ005). Moreover, financial sponsorship and also the on-site coordination work in this landmark project from Wuhan Metro Group Co., Ltd. and Shanghai Tunnel Engineering Co., Ltd. are sincerely acknowledged.

\section{References}

[1] B. Liu, R. Wang, G. Zhao et al., "Prediction of rock mass parameters in the TBM tunnel based on BP neural network integrated simulated annealing algorithm," Tunnelling and Underground Space Technology, vol. 95, 2020.

[2] Q. Pan and D. Dias, "Probabilistic evaluation of tunnel face stability in spatially random soils using sparse polynomial chaos expansion with global sensitivity analysis," Acta Geotechnica, vol. 12, no. 6, pp. 1415-1429, 2017.

[3] N. Berthoz, D. Branque, H. Wong, and D. Subrin, “TBM soft ground interaction: experimental study on a $1 \mathrm{~g}$ reduced-scale EPBS model," Tunnelling and Underground Space Technology, vol. 72, pp. 189-209, 2018.

[4] Y. Liu, E. J. Chen, S.-T. Quek et al., "Effect of spatial variation of strength and modulus on the lateral compression response of cement-admixed clay slab," Géotechnique, vol. 65, no. 10, pp. 851-865, 2015.

[5] Y. Liu, L. Q. He, Y. J. Jiang, M. M. Sun, E. J. Chen, and F.-H. Lee, "Effect of in situ water content variation on the spatial variation of strength of deep cement-mixed clay," Géotechnique, vol. 69, no. 5, pp. 391-405, 2019.

[6] E. J. Chen, L. Ding, Y. Liu, X. Ma, and M. J. Skibniewski, "On spectral representation method and Karhunen-Loève expansion in modelling construction material properties," Archives of Civil and Mechanical Engineering, vol. 18, no. 3, pp. 768-783, 2018.

[7] E. J. Chen, Y. Liu, and F.-H. Lee, "A statistical model for the unconfined compressive strength of deep-mixed columns," Géotechnique, vol. 66, no. 5, pp. 351-365, 2016.

[8] Y. Pan, Y. Liu, and E. J. Chen, "Probabilistic investigation on defective jet-grouted cut-off wall with random geometric imperfections," Géotechnique, vol. 69, no. 5, pp. 420-433, 2018.

[9] J. Ninic, C. Koch, and J. Stascheit, "An integrated platform for design and numerical analysis of shield tunnelling processes on different levels of detail," Advances in Engineering Software, vol. 112, pp. 165-179, 2017.

[10] D. Douglas-Smith, T. Iwanaga, B. F. W. Croke, and A. J. Jakeman, "Certain trends in uncertainty and sensitivity analysis: an overview of software tools and techniques," Environmental Modelling \& Software, vol. 124, Article ID 104588, 2020.

[11] W. L. Liu and L. Y. Ding, "Global sensitivity analysis of influential parameters for excavation stability of metro tunnel," Automation in Construction, vol. 113, Article ID 103080, 2020.
[12] A. Saltelli, S. Tarantola, and K. P.-S. Chan, "A quantitative model-independent method for global sensitivity analysis of model output," Technometrics, vol. 41, no. 1, pp. 39-56, 1999.

[13] A. Saltelli, Global Sensitivity Analysis: The Primer, John Wiley, Hoboken, NJ, USA, 2008.

[14] W. L. Liu, X. G. Wu, L. M. Zhang, J. J. Zheng, and J. Y. Teng, "Global sensitivity analysis of tunnel-induced building movements by a precise metamodel," Journal of Computing in Civil Engineering, vol. 31, 2017.

[15] W. Liu, X. Wu, L. Zhang, Y. Wang, and J. Teng, "Sensitivity analysis of structural health risk in operational tunnels," Automation in Construction, vol. 94, pp. 135-153, 2018.

[16] J. Li, L. Jing, X. Zheng, P. Li, and C. Yang, "Application and outlook of information and intelligence technology for safe and efficient TBM construction," Tunnelling and Underground Space Technology, vol. 93, Article ID 103097, 2019.

[17] W. H. Zhou, H. Y. Qin, J. L. Qiu et al., "Building information modelling review with potential applications in tunnel engineering of China," Royal Society Open Science, vol. 4, 2017.

[18] W. F. Cheung, T. H. Lin, and Y. C. Lin, "A real-time construction safety monitoring system for hazardous gas integrating wireless sensor network and building information modeling technologies," Sensors, vol. 18, p. 436, 2018.

[19] J. X. Zhu, G. Wright, J. Wang, and X. Y. Wang, "A critical review of the integration of geographic information system and building information modelling at the data level," ISPRS International Journal of Geo-Information, vol. 7, no. 2, p. 66, 2018.

[20] W. Solihin, C. Eastman, Y.-C. Lee, and D.-H. Yang, “A simplified relational database schema for transformation of BIM data into a query-efficient and spatially enabled database," Automation in Construction, vol. 84, pp. 367-383, 2017.

[21] R. G. Ghanem and P. D. Spanos, Stochastic Finite Element Method: Response Statistics. Stochastic Finite Elements: A Spectral Approach, Springer, Berlin, Germany, 1991.

[22] N. Wiener, "The homogeneous chaos," American Journal of Mathematics, vol. 60, pp. 560-586, 1938.

[23] D. Xiu and G. E. Karniadakis, "The wiener--askey polynomial chaos for stochastic differential equations," SIAM Journal on Scientific Computing, vol. 24, no. 2, pp. 619-644, 2002.

[24] M. Paffrath and U. Wever, "Adapted polynomial chaos expansion for failure detection," Journal of Computational Physics, vol. 226, no. 1, pp. 263-281, 2007.

[25] V. Yaghoubi, S. Marelli, B. Sudret, and T. Abrahamsson, "Sparse polynomial chaos expansions of frequency response functions using stochastic frequency transformation," Probabilistic Engineering Mechanics, vol. 48, pp. 39-58, 2017.

[26] D. Métivier, M. Vuffray, and S. Misra, "Efficient polynomial chaos expansion for uncertainty quantification in power systems," Electric Power Systems Research, vol. 189, Article ID 106791, 2020.

[27] J. C. Helton and W. L. Oberkampf, "Alternative representations of epistemic uncertainty," Reliability Engineering \& System Safety, vol. 85, no. 1-3, pp. 1-10, 2004.

[28] B. Sudret, "Global sensitivity analysis using polynomial chaos expansions," Reliability Engineering \& System Safety, vol. 93, no. 7, pp. 964-979, 2008.

[29] A. Saltelli and P. Annoni, "How to avoid a perfunctory sensitivity analysis," Environmental Modelling \& Software, vol. 25, no. 12, pp. 1508-1517, 2010.

[30] J. Nossent, P. Elsen, and W. Bauwens, “Sobol' sensitivity analysis of a complex environmental model," Environmental Modelling \& Software, vol. 26, no. 12, pp. 1515-1525, 2011. 
[31] F. Pianosi and T. Wagener, "A simple and efficient method for global sensitivity analysis based on cumulative distribution functions," Environmental Modelling \& Software, vol. 67, pp. 1-11, 2015.

[32] V. Dubourg and B. Sudret, "Meta-model-based importance sampling for reliability sensitivity analysis," Structural Safety, vol. 49, pp. 27-36, 2014.

[33] Q. Liu and T. Homma, "A new computational method of a moment-independent uncertainty importance measure," Reliability Engineering \& System Safety, vol. 94, no. 7, pp. 1205-1211, 2009.

[34] M. Jaxa-Rozen and J. Kwakkel, “Tree-based ensemble methods for sensitivity analysis of environmental models: a performance comparison with Sobol and Morris techniques," Environmental Modelling \& Software, vol. 107, pp. 245-266, 2018.

[35] L. Zhang, X. Wu, H. Zhu, and S. M. AbouRizk, "Performing global uncertainty and sensitivity analysis from given data in tunnel construction," Journal of Computing in Civil Engineering, vol. 31, no. 6, Article ID 04017065, 2017.

[36] E. Ryan, O. Wild, A. Voulgarakis, and L. Lee, "Fast sensitivity analysis methods for computationally expensive models with multi-dimensional output," Geoscientific Model Development, vol. 11, no. 8, pp. 3131-3146, 2018.

[37] R. I. Cukier, C. M. Fortuin, K. E. Shuler, A. G. Petschek, and J. H. Schaibly, "Study of the sensitivity of coupled reaction systems to uncertainties in rate coefficients. I Theory," The Journal of Chemical Physics, vol. 59, no. 8, pp. 3873-3878, 1973.

[38] A. Saltelli, M. Ratto, S. Tarantola, and F. Campolongo, "Update 1 of: sensitivity analysis for chemical models," Chem Rev, vol. 112, 2012.

[39] S. Tarantola, D. Gatelli, and T. A. Mara, "Random balance designs for the estimation of first order global sensitivity indices," Reliability Engineering \& System Safety, vol. 91, no. 6, pp. 717-727, 2006.

[40] T. A. Mara and S. Tarantola, "Application of global sensitivity analysis of model output to building thermal simulations," Building Simulation, vol. 1, no. 4, pp. 290-302, 2008.

[41] R. K. Tripathy and I. Bilionis, "Deep UQ: learning deep neural network surrogate models for high dimensional uncertainty quantification," Journal of Computational Physics, vol. 375, pp. 565-588, 2018.

[42] F. Pianosi, F. Sarrazin, and T. Wagener, "A matlab toolbox for global sensitivity analysis," Environmental Modelling \& Software, vol. 70, pp. 80-85, 2015.

[43] R. H. Sues and M. A. Cesare, "System reliability and sensitivity factors via the MPPSS method," Probabilistic Engineering Mechanics, vol. 20, no. 2, pp. 148-157, 2005.

[44] N. Fajraoui, F. Ramasomanana, A. Younes, T. A. Mara, P. Ackerer, and A. Guadagnini, "Use of global sensitivity analysis and polynomial chaos expansion for interpretation of nonreactive transport experiments in laboratory-scale porous media," Water Resources Research, vol. 47, 2011.

[45] N. Fajraoui, T. A. Mara, A. Younes, and R. Bouhlila, "Reactive transport parameter estimation and global sensitivity analysis using sparse polynomial chaos expansion," Water, Air, and Soil Pollution, vol. 223, no. 7, pp. 4183-4197, 2012.

[46] S. M. Spiessl, D.-A. Becker, and A. Rübel, "EFAST analysis applied to a PA model for a generic HLW repository in clay," Reliability Engineering \& System Safety, vol. 107, pp. 190-204, 2012.

[47] Y. He, M. Razi, C. Forestiere, L. Dal Negro, and R. M. Kirby, "Uncertainty quantification guided robust design for nanoparticles' morphology," Computer Methods in Applied Mechanics and Engineering, vol. 336, pp. 578-593, 2018.

[48] J. R. Zhang, G. Huang, and X. M. Gou, "An optimum metamodel for safety control of operational subway tunnel during underpass shield tunneling," Structural Control and Health Monitoring, vol. 25, 2018.

[49] R. Hasanpour, J. Rostami, M. Thewes, and J. Schmitt, "Parametric study of the impacts of various geological and machine parameters on thrust force requirements for operating a single shield TBM in squeezing ground," Tunnelling and Underground Space Technology, vol. 73, pp. 252-260, 2018.

[50] J. Zou and S. Zuo, "Similarity solution for the synchronous grouting of shield tunnel under the vertical non-axisymmetric displacement boundary condition," Advances in Applied Mathematics and Mechanics, vol. 9, no. 1, pp. 205-232, 2017.

[51] W. Liu, X. Wu, L. Zhang, and Y. Wang, "Probabilistic analysis of tunneling-induced building safety assessment using a hybrid FE-copula model," Structure \& Infrastructure Engineering, vol. 14, no. 8, pp. 1065-1081, 2017.

[52] C. Zhou, H. Xu, L. Ding, L. Wei, and Y. Zhou, "Dynamic prediction for attitude and position in shield tunneling: a deep learning method," Automation in Construction, vol. 105, Article ID 102840, 2019.

[53] J. Wang, X. Li, L. Lu, and F. Fang, "Parameter sensitivity analysis of crop growth models based on the extended fourier amplitude sensitivity test method," Environmental Modelling \& Software, vol. 48, pp. 171-182, 2013.

[54] E. Vanuytrecht, D. Raes, and P. Willems, "Global sensitivity analysis of yield output from the water productivity model," Environmental Modelling \& Software, vol. 51, pp. 323-332, 2014.

[55] C. F. Ma, X. Li, and S. G. Wang, "A global sensitivity analysis of soil parameters associated with backscattering using the advanced integral equation model," IEEE Transactions on Geoscience and Remote Sensing, vol. 53, pp. 5613-5623, 2015.

[56] O. Garcia-Cabrejo and A. Valocchi, "Global sensitivity analysis for multivariate output using polynomial chaos expansion," Reliability Engineering \& System Safety, vol. 126, pp. 25-36, 2014.

[57] G. Deman, K. Konakli, B. Sudret, J. Kerrou, P. Perrochet, and H. Benabderrahmane, "Using sparse polynomial chaos expansions for the global sensitivity analysis of groundwater lifetime expectancy in a multi-layered hydrogeological model," Reliability Engineering \& System Safety, vol. 147, pp. 156-169, 2016.

[58] N. Wiener, "The homogeneous chaos," American Journal of Mathematics, vol. 60, no. 4, pp. 897-936, 1938.

[59] L. Gao, B. A. Bryan, M. Nolan, J. D. Connor, X. Song, and G. Zhao, "Robust global sensitivity analysis under deep uncertainty via scenario analysis," Environmental Modelling \& Software, vol. 76, pp. 154-166, 2016.

[60] A. Cosenza, G. Mannina, P. A. Vanrolleghem, and M. B. Neumann, "Variance-based sensitivity analysis for wastewater treatment plant modelling," Science of The Total Environment, vol. 470-471, pp. 1068-1077, 2014.

[61] E. J. Mosbaek, Economic Forecasts and Policy by H. Theil, North-Holland Pub. Co., Amsterdam, Netherlands, 1961.

[62] M. D. Mckay, R. J. Beckman, and W. J. Conover, "A comparison of three methods for selecting values of input variables in the analysis of output from a computer code," Technometrics, vol. 42, no. 1, pp. 55-61, 2000.

[63] A. Radomyski, E. Giubilato, P. Ciffroy, A. Critto, C. Brochot, and A. Marcomini, "Modelling ecological and human 
exposure to POPs in Venice lagoon - Part II: quantitative uncertainty and sensitivity analysis in coupled exposure models," Science of The Total Environment, vol. 569-570, pp. 1635-1649, 2016.

[64] W. F. Luan, L. Lu, X. Li, and C. F. Ma, "Weight determination of sustainable development indicators using a global sensitivity analysis method," Sustainability, vol. 9, no. 2, p. 303, 2017.

[65] S. Marino, I. B. Hogue, C. J. Ray, and D. E. Kirschner, "A methodology for performing global uncertainty and sensitivity analysis in systems biology," Journal of Theoretical Biology, vol. 254, no. 1, pp. 178-196, 2008.

[66] M. D. Morris, "Factorial sampling plans for preliminary computational experiments," Technometrics, vol. 33, no. 2, pp. 161-174, 1991.

[67] F. Campolongo, J. Cariboni, and A. Saltelli, "An effective screening design for sensitivity analysis of large models," Environmental Modelling \& Software, vol. 22, no. 10, pp. 1509-1518, 2007.

[68] I. M. Sobol, "Global sensitivity indices for nonlinear mathematical models and their Monte Carlo estimates," Mathematics and Computers in Simulation, vol. 55, no. 1-3, pp. 271-280, 2001.

[69] A. Saltelli and S. Tarantola, "On the relative importance of input factors in mathematical models," Journal of the American Statistical Association, vol. 97, no. 459, pp. 702-709, 2002. 